\title{
A Heterogeneous Multiattribute Group Decision-Making Method Based on Intuitionistic Triangular Fuzzy Information
}

\author{
Jun Xu $\mathbb{D}^{\mathrm{D}},{ }^{1,2}$ Jiu-Ying Dong, ${ }^{2}$ Shu-Ping Wan $\mathbb{D}^{\mathrm{D}}{ }^{3}$ De-Yan Yang $\mathbb{D}^{4},{ }^{4}$ and Yi-Feng Zeng ${ }^{5}$ \\ ${ }^{1}$ College of Modern Economics \& Management, Jiangxi University of Finance and Economics, Nanchang 330013, China \\ ${ }^{2}$ School of Statistics, Jiangxi University of Finance and Economics, Nanchang 330013, China \\ ${ }^{3}$ College of Information Technology, Jiangxi University of Finance and Economics, Nanchang 330013, China \\ ${ }^{4}$ Business School, Jiangsu Normal University, Xuzhou 221116, China \\ ${ }^{5}$ Department of Computer Science and Information Systems, Teesside University, Middlesbrough Ts13ba, UK
}

Correspondence should be addressed to Shu-Ping Wan; shupingwan@163.com and De-Yan Yang; yangdeyan027@foxmail.com

Received 4 April 2019; Accepted 16 July 2019; Published 7 August 2019

Academic Editor: Chittaranjan Hens

Copyright (c) 2019 Jun Xu et al. This is an open access article distributed under the Creative Commons Attribution License, which permits unrestricted use, distribution, and reproduction in any medium, provided the original work is properly cited.

How to aggregate decision information in heterogeneous multiattribute group decision making (HMAGDM) is vital. The aim of this paper is to develop an approach to aggregating decision data into intuitionistic triangular fuzzy numbers (ITFNs) for heterogeneous MAGDM problems with real numbers (RNs), interval numbers (INs), triangular fuzzy numbers (TFNs), trapezoidal fuzzy numbers (TrFNs), and triangular intuitionistic fuzzy number (TIFNs). Using the relative closeness of technique for order preference by similarity to ideal solution (TOPSIS) and geometry entropy method, we first present a general approach to aggregating heterogeneous information into ITFNs, which takes the group consistency of experts into account. Based on the collective intuitionistic triangular fuzzy decision matrix and extended TOPSIS, a multiple objective mathematical program is constructed to determine the optimal attribute weights. Subsequently, a new method to solve HMAGDM problems is presented based on the aforementioned discussion. A trustworthy service selection example is provided to verify the practicality and effectiveness of the proposed method.

\section{Introduction}

Group decision making (GDM) has been known as a popular method for finding the best alternative from a set of alternatives through aggregating decision information given in a group of experts, in which the evaluation of alternatives may involve multiple attributes including objective and subjective information [1-3]. Due to the limited cognition and preference of decision maker, it is hard for different attributes to use the same information format to express the evaluation. For instance, in an online seller evaluation, the service attitude of the seller is suited to be described by triangular fuzzy numbers (TFNs) since the service of the seller is generally stable, but sometimes it is excellent and sometimes bad. It is convenient to describe the shipping speed of seller with interval numbers (INs) since it is not fixed but fluctuates in a certain range. These types of GDM problems with multiple conflicting attributes whose values are given by decision makers (DMs) may be represented in the form of multiple formats, such as real numbers (RNs), INs, TFNs, trapezoidal fuzzy numbers ( $\mathrm{TrFNs}$ ), and linguistic values (LVs), called heterogeneous multiattribute group decision making (HMAGDM) problems [4].

In this recent research, HMAGDM methods have been successfully applied to various fields, such as supply chain coordination [5], business processes [6], and software quality evaluation [7-9]. The key to tackling such problems is how to fuse various types of attribute values [10]. So far, many useful and valuable methods have been developed to study the fusion process of heterogeneous information, which can be roughly classified into three main categories $[4,10]$. (1) The indirect approaches [11-14], in which the heterogeneous decision information given by DMs is converted into uniformed information by transformation methods. Wang and Cai [13] developed a generic distance-based VIKOR which can use aggregation function to convert heterogeneous 
information into a uniform nonfuzzy degree and applied it to deal with emergency supplier selection. Using transformation function, Zhang et al. [14] transformed the multigranular linguistic decision matrices (LDMs) into uniform LDMs. Then, a new optimization consensus model was constructed for 2-Rank multigranular linguistic MAGDM problems. (2) The optimization-based approaches [5, 15-17], in which the heterogeneous information is integrated by constructing different multiple objective optimization models. Dong et al. [15] proposed a new complex and dynamic HMAGDM method to deal with the differences between individual sets of attributes and heterogeneous information. Zhang et al. [16] developed a HMAGDM method with aspirations information by combining the prospect theory and a biobjective intuitionistic fuzzy programming model. Yu et al. [17] incorporated risk attitude and preference deviation of experts into the mathematical programming models to solve the HMAGDM problems with RNs, INs, Ifs, LVs, and TFNs. (3) The direct approaches [10, $18-21]$. In the direct approach, the collective decision information is obtained by aggregating standardized individual decision information. Then the heterogeneous information is transformed into some comparable preference information. Yue and Jia [10] introduced a projection measure to aggregate decision information including IFNs and IVIFs. Yue [18] proposed a direct projection-based group decisionmaking methodology with RNs and INs. To overcome the irrationality of the classical projection formulae in $\mathrm{RN}$ and IN vector settings, Yue [19] presented a normalized projection measure and applied it to solve HMAGDM problems with RNs and INs. In order to integrate heterogeneously interrelated attributes in the HMAGDM problem, Das et al. [20] develop an Atanassov's intuitionistic fuzzy extended Bonferroni mean based on a strict t-conorm. Li et al. [21] proposed a new HMAGDM method using weighted power average operator to integrate the heterogeneous decision data.

These achievements have provided the foundation of the HMAGDM problems. It is noticed that methods $[1,5-$ $8,15-21]$ are on the basis of the hypothesis that the ratings provided by DMs are completely affirmative, neglecting the judgment subjectivity; thus, the impreciseness and uncertainty of original decision information cannot be captured. Methods [10-14] turned the heterogeneous information into a unified form of linguistic terms, which are subjective and cannot measure quantitatively and intuitively the uncertainty of attribute values. Intuitionistic fuzzy (IF) sets (IFSs) [22, 23] and interval-valued intuitionistic fuzzy sets (IVIFSs) [24] can be viewed as an effective tool to describe the uncertainty and ambiguity, which has led to the wide applications of IFSs and IVIFSs [25-27]. To fill these research gaps, many practical studies have been proposed to aggregate decision data into IFSs [22-24], which can be divided into two categories: (1) the methods for aggregating RNs into intuitionistic fuzzy number (IFN) based on Golden Section idea [28, 29], Minimax Criterion [30], and statistical theory [31]; (2) the methods for aggregating RNs or INs into interval-valued intuitionistic fuzzy number (IVIFN) [1] based on Minimax Criterion [32], linear transformation [33], and mean and standard deviation [34]. However, these methods [28-34] cannot be suitable for
HMAGDM problems. More recently, Xu et al. [35] presented a general method to aggregate decision information into IFN and applied it to select cloud computing service providers wherein the assessments take the form of RNs, INs, TFNs, TrFNs, and LVs. Combined with the relative closeness in technique for order preference by similarity to ideal solution (TOPSIS) and statistical theory [36], Wan et al. [37] developed a new general method to aggregate the attribute value vector into IVIFNs and used it for HMAGDM problem with RNs, INs, TFNs, and TrFNs.

The aforementioned methods [35, 37] have made deep discussions to HMAGDM problems based on aggregating decision data into IF information, but these aggregation techniques [28-35, 37] still suffer from some deficiencies. (1) They cannot deal with more complicated attribute values represented by triangular intuitionistic fuzzy number (TIFNs) and trapezoidal intuitionistic fuzzy numbers (TrIFNs). (2) They ignore the influence of different experts in aggregation process which may lead to unreasonable results. (3) The membership degree and nonmembership degree of integrated value in $[28-35,37]$ cannot reflect the distribution characteristics of the data like normal distribution. In many real decision situations, the evaluations of decision maker are based on a number of historical feedbacks on the corresponding attribute. Studies showed that the distribution of historical feedbacks is generally close to a normal distribution when the number of feedbacks is larger. It may be effective to use TFNs to model the integrated value instead of crisp value and interval-value since TFNs contain more information and are more consistent with normal distribution characteristics. Thus, when an assessment vector is aggregated into an IFN and IVIFN, the loss of information is likely to occur. Intuitionistic triangular fuzzy numbers (ITFNs) introduced by Liu and Yuan [38], as an extension of IFSs, can express more information from different dimension decision information [39] than IFNs and IVIFNs since its prominent characteristic is that the corresponding membership degree and nonmembership degree are described by TFNs [40]. Thus, ITFNs can not only depict the fuzzy concept of "good" or "excellent," but also outstand the satisfaction and dissatisfaction information with the maximum probability and also recoup the deficiency due to the loss of the center of gravity in IVIFNs [41-43]. For instance, in a trustworthy seller selection example, the service attitude may be expressed by an ITFN $((0.4,0.6,0.7),(0.1,0.2,0.3))$, which contains two aspects of implication in the historical ratings of a seller: one is that users' satisfactory degree is between 0.4 and 0.7 ; the most possible satisfactory degree is 0.6 ; the other is that users' dissatisfactory degree is between 0.1 and 0.3 ; the most possible dissatisfactory degree is 0.2 . Some theories and GDM methods based on ITFNs have been developed. Wang [40] defined score function and accuracy function to compare the ITFNs and developed several ITFN geometric aggregation operators. Wei [41] proposed the ITFN weighted averaging operator and ITFN ordered weighted averaging operator and applied them to solve GDM problems. To consider the interaction among attributes, Gao et al. [42] presented some ITFN aggregation operators with interaction. Yu and $\mathrm{Xu}$ [43] investigated a series of intuitionistic multiplicative triangular 
fuzzy aggregation operators. Although these studies [3843] focused on different aspects of ITFNs, it can only aggregate ITFNs. Therefore, to push ahead with the application of the above aggregations, it is necessary to aggregate multiple types of decision information into ITFNs, which is very interesting yet relatively sophisticated to dispose of.

To do that, this paper aims to propose a novel HMAGDM method based on ITFNs. The primary contributions of this paper can be illuminated briefly as follows.

(1) We first present an aggregation technology to aggregate heterogeneous information into TIFNs. Compared with existing methods $[28-35,37]$, the proposed aggregate technology has the following advantages. For more details, refer to Section 5.2.

(i) A new elicitation of the support, opposite, and uncertain information based on distance is introduced, which can accommodate more complicated attribute values including TIFNs and TrIFNs since it just needs to calculate the distance from decision data to the maximum and minimum grade.

(ii) A new construction approach of ITFN is presented by group consistency which not only takes into account expert's weight but can overcome the shortcoming of the hypothesis of the normal distribution.

(iii) It can not only effectively avoid the loss of original information, but also reflect the distribution characteristics of the original decision data.

(2) A new similarity measure of ITFNs is developed and applied to construct a multiple objective linear programming to determine attribute weights in ITFN environment with incomplete information. The determination method of attribute weights can effectively avoid the subjectivity brought by the given attribute weights in advance.

(3) Based on the aforesaid provision, a new method to deal with HMAGDM problems with RNs, INs, TFNs, TrFNs, and TIFNs is proposed. The comprehensive evaluation value of the alternative is an ITFN, which preserves more useful information.

The remainder of this paper is set out as follows. Section 2 briefly introduces related basic concepts. Section 3 presents an approach to aggregating heterogeneous decision data into ITFNs. Section 4 builds a multiple objective linear programming model to determine attribute weights and propose a HMAGDM method. Section 5 provides a numerical example to illustrate the feasibility and reasonableness of the proposed method. Section 6 makes our conclusions.

\section{Preliminary}

In this section, some basic concepts of ITFN and distance measures are briefly described below.

\subsection{Intuitionistic Triangular Fuzzy Number}

Definition 1 (see [38]). A triangular fuzzy number (TFN) $A$ is a special fuzzy set on a real number set $R$; its membership function is defined by

$$
F_{A}(x)= \begin{cases}\frac{x-t_{l}}{t_{m}-t_{l}}, & \text { if } t_{l} \leq x \leq t_{m}, \\ \frac{t_{h}-x}{t_{h}-t_{m}}, & \text { if } t_{m} \leq x \leq t_{h}, \\ 0, & \text { if otherwise, }\end{cases}
$$

where $0 \leq t_{l} \leq t_{m} \leq t_{h} \leq 1, t_{l}$ and $t_{h}$ present the lower limit and upper limit of $A$, respectively, and $t_{m}$ is the mode, which can be denoted as a triplet $\left(t_{l}, t_{m}, t_{h}\right)$.

Definition 2 (see [38]). Let $X$ be a fixed set; $\mu_{\widetilde{A}}(x)=$ $\left(t_{A}^{l}(x), t_{A}^{m}(x), t_{A}^{h}(x)\right)$ and $v_{\widetilde{A}}(x)=\left(f_{A}^{l}(x), f_{A}^{m}(x), f_{A}^{h}(x)\right)$ are TFNs defined on the unit interval $[0,1]$; then an intuitionistic triangular fuzzy set $\widetilde{A}$ over $X$ is defined as $\widetilde{A}=\{(x,<$ $\left.\left.\mu_{\widetilde{A}}(x), v_{\widetilde{A}}(x)>\right) \mid x \in X\right\}$ where the parameters $\mu_{\widetilde{A}}(x)$ and $v_{\widetilde{A}}(x)$ indicate, respectively, the membership degree and nonmembership degree of the element $x$ in $\widetilde{A}$, with the conditions $0 \leq t_{A}^{h}(x)+f_{A}^{h}(x) \leq 1$.

For convenience, we call $\widetilde{\alpha}=\left(\left(t_{A}^{l}, t_{A}^{m}, t_{A}^{h}\right),\left(f_{A}^{l}, f_{A}^{m}, f_{A}^{h}\right)\right)$ an intuitionistic triangular fuzzy number (ITFN), where

$$
\begin{array}{r}
t_{A}^{l}, t_{A}^{m}, t_{A}^{h} \in[0,1], \\
f_{A}^{l}, f_{A}^{m}, f_{A}^{h} \in[0,1], \\
t_{A}^{h}+f_{A}^{h} \in[0,1] .
\end{array}
$$

It is clear that the largest and smallest ITFN are $\alpha^{+}=$ $((1,1,1),(0,0,0))$ and $\alpha^{-}=((0,0,0),(1,1,1))$, respectively.

Definition 3 (see [38]). Let $\widetilde{\alpha}_{1}=\left(\left(t_{1}^{l}, t_{1}^{m}, t_{1}^{h}\right),\left(f_{1}^{l}, f_{1}^{m}, f_{1}^{h}\right)\right)$ and $\widetilde{\alpha}_{2}=\left(\left(t_{2}^{l}, t_{2}^{m}, t_{2}^{h}\right),\left(f_{2}^{l}, f_{2}^{m}, f_{2}^{h}\right)\right)$ be two ITFNs; then the containment is

$$
\begin{aligned}
& \widetilde{\alpha}_{1} \subseteq \widetilde{\alpha}_{2} \\
& \text { iff } t_{1}^{l} \leq t_{2}^{l}, t_{1}^{m} \leq t_{2}^{m}, t_{1}^{h} \leq t_{2}^{h}, f_{1}^{l} \geq f_{2}^{l}, f_{1}^{m} \geq f_{2}^{m} \text { and } f_{1}^{h} \geq f_{2}^{h} .
\end{aligned}
$$

Some arithmetic operations between ITFNs $\widetilde{\alpha}_{1}$ and $\widetilde{\alpha}_{2}$ are shown as below [40]:

(1) $\widetilde{\alpha}_{1}+\widetilde{\alpha}_{2}=\left(\left(t_{1}^{l}+t_{2}^{l}-t_{1}^{l} t_{2}^{l}, t_{1}^{m}+t_{2}^{m}-t_{1}^{m} t_{2}^{m}, t_{1}^{h}+t_{2}^{h}-t_{1}^{h} t_{2}^{h}\right)\right.$, $\left.\left(f_{1}^{l} f_{2}^{l}, f_{1}^{m} f_{2}^{m}, f_{1}^{h} f_{2}^{h}\right)\right)$,

(2) $\lambda \widetilde{\alpha}=\left(\left(1-\left(1-t_{1}^{l}\right)^{\lambda}, 1-\left(1-t_{1}^{m}\right)^{\lambda}, 1-\left(1-t_{1}^{h}\right)^{\lambda}\right)\right.$, $\left.\left(\left(f_{1}^{l}\right)^{\lambda},\left(f_{1}^{m}\right)^{\lambda},\left(f_{1}^{h}\right)^{\lambda}\right)\right) \lambda>0$. 
Definition 4 (see [41]). For a set of ITFNs $\widetilde{\alpha}_{i}(i=1,2, \ldots, n)$ that have associated an importance weight vector $\boldsymbol{w}=$ $\left(w_{1}, w_{2}, \ldots, w_{n}\right)^{T}$ with $w_{i} \in[0,1]$ and $\sum_{i=1}^{n} w_{i}=1$. We call

$$
\begin{aligned}
& \operatorname{ITFWA}\left(\tilde{a}_{1}, \tilde{a}_{2}, \ldots, \tilde{a}_{n}\right)=\sum_{i=1}^{n} w_{i} \tilde{a}_{i}=((1 \\
& -\prod_{i=1}^{n}\left(1-t_{i}^{l}\right)^{w_{i}}, 1-\prod_{i=1}^{n}\left(1-t_{i}^{m}\right)^{w_{i}}, 1 \\
& \left.-\prod_{i=1}^{n}\left(1-t_{i}^{h}\right)^{w_{i}}\right), \\
& \left.\left(\prod_{i=1}^{n}\left(f_{i}^{l}\right)^{w_{i}}, \prod_{i=1}^{n}\left(f_{i}^{m}\right)^{w_{i}}, \prod_{i=1}^{n}\left(f_{i}^{h}\right)^{w_{i}}\right)\right)
\end{aligned}
$$

an intuitionistic triangular fuzzy weighted average operator (ITFWA).

Definition 5. Let $\widetilde{\alpha}_{1}=\left(\left(t_{1}^{l}, t_{1}^{m}, t_{1}^{h}\right),\left(f_{1}^{l}, f_{1}^{m}, f_{1}^{h}\right)\right)$ and $\widetilde{\alpha}_{2}=$ $\left(\left(t_{2}^{l}, t_{2}^{m}, t_{2}^{h}\right),\left(f_{2}^{l}, f_{2}^{m}, f_{2}^{h}\right)\right)$ be two ITFNs. A similarity measure $\vartheta\left(\widetilde{\alpha}_{1}, \widetilde{\alpha}_{2}\right)$ between the ITFNs $\widetilde{\alpha}_{1}$ and $\widetilde{\alpha}_{2}$ is defined as follows:

$$
\begin{aligned}
& \vartheta\left(\widetilde{\alpha}_{1}, \widetilde{\alpha}_{2}\right)=1-\left[\frac { 1 } { 1 2 } \left(\left|t_{1}^{l}-t_{2}^{l}\right|+\left|t_{1}^{m}-t_{2}^{m}\right|+\left|t_{1}^{h}-t_{2}^{h}\right|\right.\right. \\
& \left.+\left|f_{1}^{l}-f_{2}^{l}\right|+\left|f_{1}^{m}-f_{2}^{m}\right|+\left|f_{1}^{h}-f_{2}^{h}\right|\right)+\frac{1}{2} \\
& \cdot \max \left(\left|t_{1}^{l}-t_{2}^{l}\right|,\left|t_{1}^{m}-t_{2}^{m}\right|,\left|t_{1}^{h}-t_{2}^{h}\right|,\left|f_{1}^{l}-f_{2}^{l}\right|,\right. \\
& \left.\left.\left|f_{1}^{m}-f_{2}^{m}\right|,\left|f_{1}^{h}-f_{2}^{h}\right|\right)\right]
\end{aligned}
$$

Theorem 6. The similarity measure $\vartheta\left(\widetilde{\alpha}_{1}, \widetilde{\alpha}_{2}\right)$ satisfies the following properties:

(i) $0 \leq \vartheta\left(\widetilde{\alpha}_{1}, \widetilde{\alpha}_{2}\right) \leq 1$.

(ii) $\vartheta\left(\widetilde{\alpha}_{1}, \widetilde{\alpha}_{2}\right)=1$ if and only if $\widetilde{\alpha}_{1}=\widetilde{\alpha}_{2}$.

(iii) $\vartheta\left(\widetilde{\alpha}_{1}, \widetilde{\alpha}_{2}\right)=\vartheta\left(\widetilde{\alpha}_{2}, \widetilde{\alpha}_{1}\right)$.

(iv) If $\widetilde{\alpha}_{3}$ is a ITFN and $\widetilde{\alpha}_{1} \subseteq \widetilde{\alpha}_{2} \subseteq \widetilde{\alpha}_{3}$, then $\vartheta\left(\widetilde{\alpha}_{1}, \widetilde{\alpha}_{3}\right) \leq$ $\vartheta\left(\widetilde{\alpha}_{1}, \widetilde{\alpha}_{2}\right)$ and $\vartheta\left(\widetilde{\alpha}_{1}, \widetilde{\alpha}_{3}\right) \leq \vartheta\left(\widetilde{\alpha}_{2}, \widetilde{\alpha}_{3}\right)$.

Proof. It is easy to see that the proposed similarity measure $\vartheta\left(\widetilde{\alpha}_{1}, \widetilde{\alpha}_{2}\right)$ meets the third property of Theorem 6 . We only need to prove (i), (ii), and (iv).

For (i). By (2), we have

$$
\begin{aligned}
& 0 \leq\left|t_{1}^{l}-t_{2}^{l}\right| \leq 1, \\
& 0 \leq\left|t_{1}^{m}-t_{2}^{m}\right| \leq 1, \\
& 0 \leq\left|t_{1}^{h}-t_{2}^{h}\right| \leq 1, \\
& 0 \leq\left|f_{1}^{l}-f_{2}^{l}\right| \leq 1,
\end{aligned}
$$

$$
\begin{aligned}
& 0 \leq\left|f_{1}^{m}-f_{2}^{m}\right| \leq 1, \\
& 0 \leq\left|f_{1}^{h}-f_{2}^{h}\right| \leq 1 .
\end{aligned}
$$

It is easy to see that

$$
\begin{aligned}
0 \leq & \frac{1}{12}\left(\left|t_{1}^{l}-t_{2}^{l}\right|+\left|t_{1}^{m}-t_{2}^{m}\right|+\left|t_{1}^{h}-t_{2}^{h}\right|+\left|f_{1}^{l}-f_{2}^{l}\right|\right. \\
& \left.+\left|f_{1}^{m}-f_{2}^{m}\right|+\left|f_{1}^{h}-f_{2}^{h}\right|\right) \leq \frac{1}{2} \\
0 \leq & \frac{1}{2} \max \left(\left|t_{1}^{l}-t_{2}^{l}\right|,\left|t_{1}^{m}-t_{2}^{m}\right|,\left|t_{1}^{h}-t_{2}^{h}\right|,\left|f_{1}^{l}-f_{2}^{l}\right|,\right. \\
& \left.\left|f_{1}^{m}-f_{2}^{m}\right|,\left|f_{1}^{h}-f_{2}^{h}\right|\right) \leq \frac{1}{2}
\end{aligned}
$$

Thus we get

$$
\begin{aligned}
0 & \leq 1-\left[\frac { 1 } { 1 2 } \left(\left|t_{1}^{l}-t_{2}^{l}\right|+\left|t_{1}^{m}-t_{2}^{m}\right|+\left|t_{1}^{h}-t_{2}^{h}\right|+\mid f_{1}^{l}\right.\right. \\
& \left.-f_{2}^{l}|+| f_{1}^{m}-f_{2}^{m}|+| f_{1}^{h}-f_{2}^{h} \mid\right)+\frac{1}{2} \max \left(\left|t_{1}^{l}-t_{2}^{l}\right|,\right. \\
& \left.\left.\left|t_{1}^{m}-t_{2}^{m}\right|,\left|t_{1}^{h}-t_{2}^{h}\right|,\left|f_{1}^{l}-f_{2}^{l}\right|,\left|f_{1}^{m}-f_{2}^{m}\right|,\left|f_{1}^{h}-f_{2}^{h}\right|\right)\right] \\
& \leq 1
\end{aligned}
$$

And then the inequality $0 \leq \vartheta\left(\widetilde{\alpha}_{1}, \widetilde{\alpha}_{2}\right) \leq 1$ is established. For (ii). When $\vartheta\left(\widetilde{\alpha}_{1}, \widetilde{\alpha}_{2}\right)=1$, if and only if

$$
\begin{aligned}
& \frac{1}{12}\left(\left|t_{1}^{l}-t_{2}^{l}\right|+\left|t_{1}^{m}-t_{2}^{m}\right|+\left|t_{1}^{h}-t_{2}^{h}\right|+\left|f_{1}^{l}-f_{2}^{l}\right|+\mid f_{1}^{m}\right. \\
& \left.\quad-f_{2}^{m}|+| f_{1}^{h}-f_{2}^{h} \mid\right)=0 \\
& \frac{1}{2} \max \left(\left|t_{1}^{l}-t_{2}^{l}\right|,\left|t_{1}^{m}-t_{2}^{m}\right|,\left|t_{1}^{h}-t_{2}^{h}\right|,\left|f_{1}^{l}-f_{2}^{l}\right|,\left|f_{1}^{m}-f_{2}^{m}\right|,\right. \\
& \left.\quad\left|f_{1}^{h}-f_{2}^{h}\right|\right)=0
\end{aligned}
$$

Apparently, it is easy to derive

$$
\begin{aligned}
\left|t_{1}^{l}-t_{2}^{l}\right| & =0, \\
\left|t_{1}^{m}-t_{2}^{m}\right| & =0, \\
\left|t_{1}^{h}-t_{2}^{h}\right| & =0, \\
\left|f_{1}^{l}-f_{2}^{l}\right| & =0, \\
\left|f_{1}^{m}-f_{2}^{m}\right| & =0, \\
\left|f_{1}^{h}-f_{2}^{h}\right| & =0 .
\end{aligned}
$$

Thus we get $t_{1}^{l}=t_{2}^{l}, t_{1}^{m}=t_{2}^{m}, t_{1}^{h}=t_{2}^{h}, f_{1}^{l}=f_{2}^{l}, f_{1}^{m}=f_{2}^{m}$, $f_{1}^{h}-f_{2}^{h}$. And then $\widetilde{\alpha}_{1}=\tilde{\alpha}_{2}$. 
For (iv). Since

$$
\begin{aligned}
& t_{1}^{l} \leq t_{2}^{l} \leq t_{3}^{l}, \\
& t_{1}^{m} \leq t_{2}^{m} \leq t_{3}^{m}, \\
& t_{1}^{h} \leq t_{2}^{h} \leq t_{3}^{h}, \\
& f_{1}^{l} \geq f_{2}^{l} \geq f_{3}^{l}, \\
& f_{1}^{m} \geq f_{2}^{m} \geq f_{3}^{m}, \\
& f_{1}^{h} \geq f_{2}^{h} \geq f_{3}^{h},
\end{aligned}
$$

we get

$$
\begin{aligned}
\left|t_{1}^{l}-t_{2}^{l}\right| & \leq\left|t_{1}^{l}-t_{3}^{l}\right|, \\
\left|t_{1}^{m}-t_{2}^{m}\right| & \leq\left|t_{1}^{m}-t_{3}^{m}\right|, \\
\left|t_{1}^{h}-t_{2}^{h}\right| & \leq\left|t_{1}^{h}-t_{3}^{h}\right|, \\
\left|f_{1}^{l}-f_{2}^{l}\right| & \leq\left|f_{1}^{l}-f_{3}^{l}\right|, \\
\left|f_{1}^{m}-f_{2}^{m}\right| & \leq\left|f_{1}^{m}-f_{3}^{m}\right|, \\
\left|f_{1}^{h}-f_{2}^{h}\right| & \leq\left|f_{1}^{h}-f_{3}^{h}\right| .
\end{aligned}
$$

Based on the above inequalities, it is easy to derive

$$
\begin{aligned}
& \left|t_{1}^{l}-t_{2}^{l}\right|+\left|t_{1}^{m}-t_{2}^{m}\right|+\left|t_{1}^{h}-t_{2}^{h}\right|+\left|f_{1}^{l}-f_{2}^{l}\right|+\mid f_{1}^{m} \\
& \quad-f_{2}^{m}|+| f_{1}^{h}-f_{2}^{h}|\leq| t_{1}^{l}-t_{3}^{l}|+| t_{1}^{m}-t_{3}^{m}|+| t_{1}^{h}-t_{3}^{h} \mid \\
& \quad+\left|f_{1}^{l}-f_{3}^{l}\right|+\left|f_{1}^{m}-f_{3}^{m}\right|+\left|f_{1}^{h}-f_{3}^{h}\right|,
\end{aligned}
$$

$$
\begin{aligned}
& \max \left(\left|t_{1}^{l}-t_{2}^{l}\right|,\left|t_{1}^{m}-t_{2}^{m}\right|,\left|t_{1}^{h}-t_{2}^{h}\right|,\left|f_{1}^{l}-f_{2}^{l}\right|,\left|f_{1}^{m}-f_{2}^{m}\right|,\right. \\
& \left.\left|f_{1}^{h}-f_{2}^{h}\right|\right) \leq \max \left(\left|t_{1}^{l}-t_{3}^{l}\right|,\left|t_{1}^{m}-t_{3}^{m}\right|,\left|t_{1}^{h}-t_{3}^{h}\right|,\right. \\
& \left.\left|f_{1}^{l}-f_{3}^{l}\right|,\left|f_{1}^{m}-f_{3}^{m}\right|,\left|f_{1}^{h}-f_{3}^{h}\right|\right) .
\end{aligned}
$$

Thus, it holds that

$$
\begin{aligned}
1 & -\left[\frac { 1 } { 1 2 } \left(\left|t_{1}^{l}-t_{2}^{l}\right|+\left|t_{1}^{m}-t_{2}^{m}\right|+\left|t_{1}^{h}-t_{2}^{h}\right|+\left|f_{1}^{l}-f_{2}^{l}\right|\right.\right. \\
& \left.+\left|f_{1}^{m}-f_{2}^{m}\right|+\left|f_{1}^{h}-f_{2}^{h}\right|\right)+\frac{1}{2} \max \left(\left|t_{1}^{l}-t_{2}^{l}\right|,\right. \\
& \left.\left.\left|t_{1}^{m}-t_{2}^{m}\right|,\left|t_{1}^{h}-t_{2}^{h}\right|,\left|f_{1}^{l}-f_{2}^{l}\right|,\left|f_{1}^{m}-f_{2}^{m}\right|,\left|f_{1}^{h}-f_{2}^{h}\right|\right)\right] \\
& \geq 1-\left[\frac { 1 } { 1 2 } \left(\left|t_{1}^{l}-t_{2}^{l}\right|+\left|t_{1}^{m}-t_{2}^{m}\right|+\left|t_{1}^{h}-t_{2}^{h}\right|+\mid f_{1}^{l}\right.\right. \\
& \left.-f_{2}^{l}|+| f_{1}^{m}-f_{2}^{m}|+| f_{1}^{h}-f_{2}^{h} \mid\right)+\frac{1}{2} \max \left(\left|t_{1}^{l}-t_{2}^{l}\right|,\right. \\
& \left.\left.\left|t_{1}^{m}-t_{2}^{m}\right|,\left|t_{1}^{h}-t_{2}^{h}\right|,\left|f_{1}^{l}-f_{2}^{l}\right|,\left|f_{1}^{m}-f_{2}^{m}\right|,\left|f_{1}^{h}-f_{2}^{h}\right|\right)\right] .
\end{aligned}
$$

Thus, $\vartheta\left(\widetilde{\alpha}_{1}, \widetilde{\alpha}_{3}\right) \leq \vartheta\left(\widetilde{\alpha}_{1}, \widetilde{\alpha}_{2}\right)$. In the same way, it is proved that $\vartheta\left(\widetilde{\alpha}_{1}, \widetilde{\alpha}_{3}\right) \leq \vartheta\left(\widetilde{\alpha}_{2}, \widetilde{\alpha}_{3}\right)$.

2.2. Distance Measures. Hamming distance is easily processed and commonly used in the process of heterogeneous information processing. For INs $a=\left[a^{l}, a^{u}\right]$ and $b=$ $\left[b^{l}, b^{u}\right]$ (or TFNs $a=\left(a_{1}, a_{2}, a_{3}\right)$ and $b=\left(b_{1}, b_{2}, b_{3}\right)$, TrFNs $a=\left(a_{1}, a_{2}, a_{3}, a_{4}\right)$ and $b=\left(b_{1}, b_{2}, b_{3}, b_{4}\right)$, and TIFNs $a=$ $\left(\left(a_{1}, a_{2}, a_{3}\right), u_{a}, v_{a}\right)$ and $\left.b=\left(\left(b_{1}, b_{2}, b_{3}\right), u_{b}, v_{b}\right)\right)$, the distance measures can be defined as follows $[35,44]$ :

$$
\begin{aligned}
& d(a, b) \\
& =\left\{\begin{array}{l}
\frac{1}{2}\left(\left|a^{l}-b^{l}\right|+\left|a^{u}-b^{u}\right|\right), \\
\frac{1}{3}\left(\left|a_{1}-b_{1}\right|+\left|a_{2}-b_{2}\right|+\left|a_{3}-b_{3}\right|\right), \\
\frac{1}{4}\left(\left|a_{1}-b_{1}\right|+\left|a_{2}-b_{2}\right|+\left|a_{3}-b_{3}\right|+\left|a_{4}-b_{4}\right|\right), \\
\frac{1}{6}\left(\left|u_{a} a_{1}-u_{b} b_{1}\right|+\left|u_{a} a_{2}-u_{b} b_{2}\right|+\left|u_{a} a_{3}-u_{b} b_{3}\right|\right)+\mid v_{a} a_{1}
\end{array}\right. \\
& \text { 3. A New Method for Heterogeneous } \\
& \text { MAGDM Problems }
\end{aligned}
$$

In this section, the presentation of heterogeneous MAGDM problems is given first. Then, an approach to aggregating heterogeneous information into ITFNs is developed.

3.1. Heterogeneous MAGDM Problems. For the sake of convenience, some symbols are introduced to characterize the heterogeneous MAGDM problems as follows:

(1) The group of DMs $D_{i}(i \in M=\{1,2, \ldots, m\})$.

(2) The set of attributes $A_{j}(j \in N=\{1,2, \ldots, n\})$. Denote the attribute weight vector by $\boldsymbol{w}=\left(w_{1}, w_{2}, \ldots, w_{n}\right)$, where $w_{j}$ represents the weight of $A_{j}$ such that $w_{j} \in[0,1](j \in N)$ and $\sum_{j=1}^{n} w_{j}=1$.

(3) The set of alternatives $S_{k}(k \in P=\{1,2, \ldots, p\})$.

Since there are multiple formats of rating values, the attribute set $\mathrm{A}=\left\{A_{1}, A_{2}, \ldots, A_{n}\right\}$ is divided into four subsets $\widehat{A}_{1}=\left\{A_{1}, A_{2}, \ldots, A_{j_{1}}\right\}, \widehat{A}_{2}=\left\{A_{j_{1}+1}, A_{j_{1}+2}, \ldots, A_{j_{2}}\right\}, \widehat{A}_{3}=$ $\left\{A_{j_{2}+1}, A_{j_{2}+2}, \ldots, A_{j_{3}}\right\}, \widehat{A}_{4}=\left\{A_{j_{3}+1}, A_{j_{3}+2}, \ldots, A_{j_{4}}\right\}$, and $\widehat{A}_{5}=\left\{A_{j_{4}+1}, A_{j_{4}+2}, \ldots, A_{j_{5}}\right\}$, where $1 \leq j_{1} \leq j_{2} \leq j_{3} \leq$ $j_{4} \leq j_{5} \leq n, \widehat{A}_{t} \cap \widehat{A}_{k}=\emptyset(t, k=1,2,3,4,5 ; t \neq k)$, and $\bigcup_{t=1}^{5} \widehat{A}_{t}=A, \emptyset$ is an empty set. The rating values in the subsets $\widehat{A}_{e}(e=1,2,3,4,5)$ are in the form of RNs, INs, TFNs, TrFNs, and TIFNs, respectively. Denote the subscript 


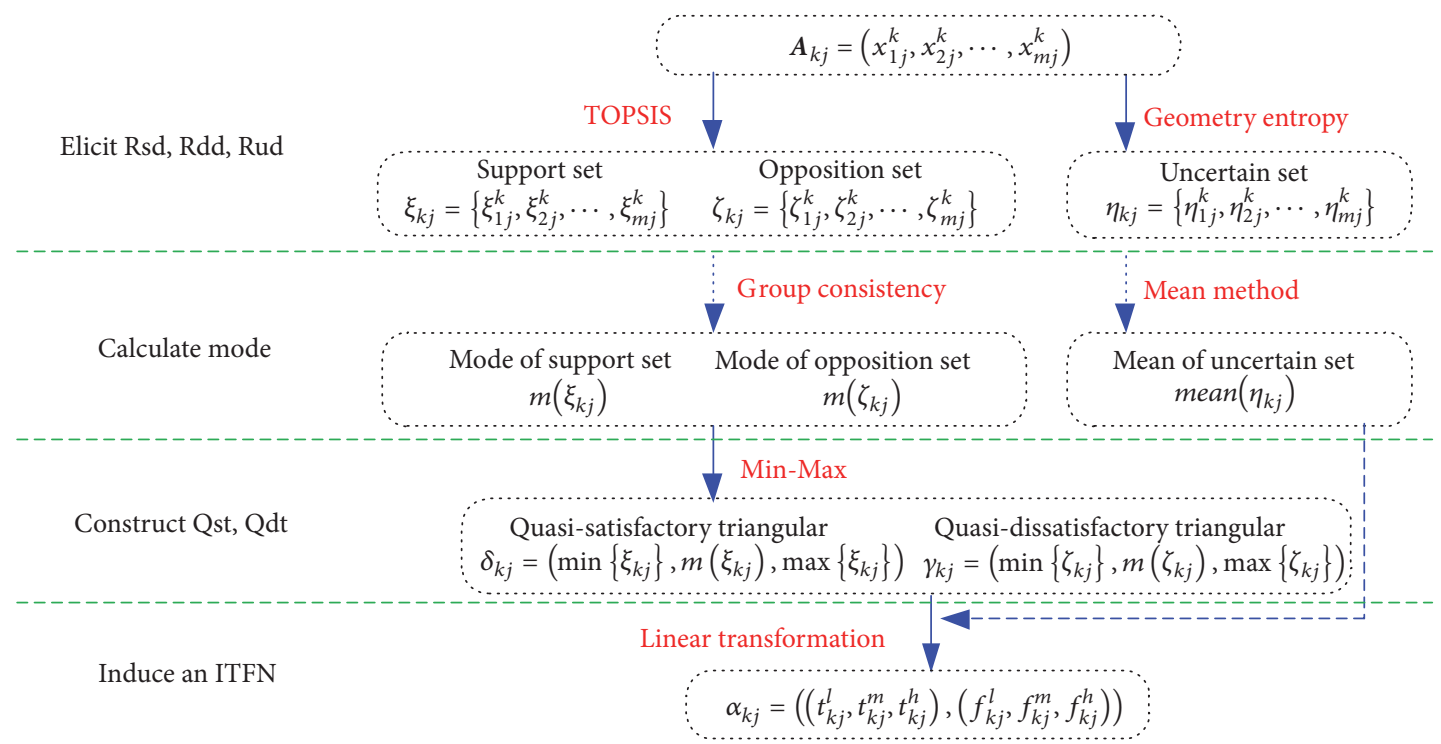

FIGURE 1: Framework for aggregating ITFN.

sets for subsets $\widehat{A}_{e}(e=1,2,3,4,5)$ by $N_{1}=\left\{1,2, \ldots, j_{1}\right\}$, $N_{2}=\left\{j_{1}+1, j_{1}+2, \ldots, j_{2}\right\}, N_{3}=\left\{j_{2}+1, j_{2}+2, \ldots, j_{3}\right\}$, $N_{4}=\left\{j_{3}+1, j_{3}+2, \ldots, j_{4}\right\}$, and $N_{5}=\left\{j_{4}+1, j_{4}+2, \ldots, j_{5}\right\}$, respectively.

(4) The group decision matrix.

Suppose that the rating of alternative $S_{k}$ with respect to the attribute $A_{j}$ given by DM $D_{i}$ is denoted by $x_{i j}^{k}(i \in M, j \in$ $N, k \in P)$. If $j \in N_{1}$, then $x_{i j}^{k}$ is a RN. If $j \in N_{2}$, then $x_{i j}^{k}=$ $\left[\underline{x}_{i j}^{k}, \bar{x}_{i j}^{k}\right]$ is an IN. If $j \in N_{3}$, then $x_{i j}^{k}=\left(a_{i j}^{k}, b_{i j}^{k}, c_{i j}^{k}\right)$ is a TFN. If $j \in N_{4}$, then $x_{i j}^{k}=\left(e_{i j}^{k}, f_{i j}^{k}, g_{i j}^{k}, h_{i j}^{k}\right)$ is a TrFN. If $j \in N_{5}$, then $x_{i j}^{k}=\left(\left(\underline{t}_{i j}^{k}, t_{i j}^{k}, \bar{t}_{i j}^{k}\right), u_{i j}^{k}, v_{i j}^{k}\right)$. Namely, $x_{i j}^{k}$ can be unified as follows:

$$
x_{i j}^{k}= \begin{cases}x_{i j}^{k}, & \text { if } j \in N_{1} \\ {\left[\underline{x}_{i j}^{k}, \bar{x}_{i j}^{k}\right],} & \text { if } j \in N_{2} \\ \left(a_{i j}^{k}, b_{i j}^{k}, c_{i j}^{k}\right), & \text { if } j \in N_{3} \\ \left(e_{i j}^{k}, f_{i j}^{k}, g_{i j}^{k}, h_{i j}^{k}\right), & \text { if } j \in N_{4} \\ \left(\left(\underline{t}_{i j}^{k}, t_{i j}^{k}, \bar{t}_{i j}^{k}\right), u_{i j}^{k}, v_{i j}^{k}\right), & \text { if } j \in N_{5}\end{cases}
$$

Hence, a group decision matrix of alternative $S_{k}$ can be expressed as

$$
\boldsymbol{X}^{k}=\left(x_{i j}^{k}\right)_{m \times n}=\begin{gathered}
A_{1} \\
D_{1} \\
D_{2} \\
\vdots \\
D_{m}
\end{gathered}\left(\begin{array}{cccc}
x_{11}^{k} & x_{12}^{k} & \cdots & x_{1 n}^{k} \\
x_{21}^{k} & x_{22}^{k} & \cdots & x_{2 n}^{k} \\
\vdots & \vdots & \vdots & \vdots \\
x_{m 1}^{k} & x_{m 2}^{k} & \cdots & x_{m n}^{k}
\end{array}\right)
$$

To reduce information loss and simplify the focused problems, the group decision matrices $\boldsymbol{X}^{k}=\left(x_{i j}^{k}\right)_{m \times n}(k=$ $1,2, \ldots, p)$ can be integrated into a collective ITFN decision matrix. The key to addressing this issue lies in an effective approach for constructing ITFNs based on the experts' assessment expressed in different types of data.

3.2. An Approach to Aggregating Heterogeneous Information into ITFNs. To facilitate the calculation, denote the $j$ th column vector in the matrix $\boldsymbol{X}^{k}$ as

$$
\boldsymbol{A}_{k j}=\left(x_{1 j}^{k}, x_{2 j}^{k}, \ldots, x_{m j}^{k}\right) \quad(k \in P, j \in N),
$$

which is the normalized assessment vector of alternative $S_{k}$ on attribute $A_{j}$ given by all DMs $D_{i}(i=1,2, \ldots, m)$. Let $A_{j}^{\max }$ and $A_{j}^{\mathrm{min}}$ be the largest grade and smallest grade employed in the rating system. For example, if the assessments in $A_{j}$ are TFNs, then $A_{j}^{\max }=(1,1,1)$ and $A_{j}^{\min }=(0,0,0)$; if the assessments in $A_{j}$ are INs, then $A_{j}^{\max }=[1,1]$ and $A_{j}^{\min }=$ $[0,0]$. To integrate the decision matrices $\boldsymbol{X}^{k}=\left(x_{i j}^{k}\right)_{m \times n}(k=$ $1,2, \ldots, p)$ into a collective ITFN decision matrix, all the elements in vector $\boldsymbol{A}_{k j}$ need to be aggregated into an ITFN. The implementation of the aggregation approach involves a four-stage framework (see Figure 1): (1) Elicit Rsd, Rdd, and Rud. In this process, we use the TOPSIS method to obtain the rating satisfactory degree (Rsd) and rating dissatisfactory degree (Rdd) of $x_{i j}^{k}$ and construct the support set $\xi_{k j}$ and opposition set $\zeta_{k j}$ of $\boldsymbol{A}_{k j}$. The rating uncertain degree (Rud) of $x_{i j}^{k}$ and the corresponding uncertain set $\eta_{k j}$ are derived by geometry entropy. (2) Calculate mode. Combining the group consistency and mean method, the modes of the above sets $\xi_{k j}, \zeta_{k j}$, and $\eta_{k j}$ are computed in this stage. (3) Construct Qst and Qdt. According to the Min-Max method, the quasisatisfactory triangular (Qst) and quasi-dissatisfactory triangular (Qdt) of $\boldsymbol{A}_{k j}$ can be built. (4) Induce an ITFN. The ITFN 


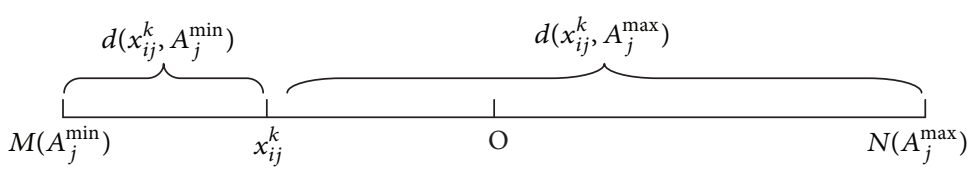

FIGURE 2: Distances ratio-based rating uncertain degree for $x_{i j}^{k}$.

of $\boldsymbol{A}_{k j}$ can be obtained through a linear transformation in this process.

3.2.1. Elicit the Rsd, Rdd, and Rud. Consider that (1) the relative closeness [45] from $x_{i j}^{k}$ to $A_{j}^{\max }$ implies the satisfaction of DM; (2) the relative closeness from $x_{i j}^{k}$ to $A_{j}^{\mathrm{min}}$ implies the dissatisfaction of DM; and (3) according to the ratiobased measure of fuzziness [46, 47], the ratio of distances from $x_{i j}^{k}$ to $A_{j}^{\min }$ and from $x_{i j}^{k}$ to $A_{j}^{\max }$ can also express the fuzziness degree of $x_{i j}^{k}$. Thus, combining the relative closeness of TOPSIS [36] and geometry entropy method [46], the Rsd, Rdd, and Rud of $x_{i j}^{k}$ can be elicited as follows.

Definition 7. Let $\boldsymbol{A}_{k j}$ be a benefit attribute vector, and let $x_{i j}^{k}$ be an arbitrary element in $\boldsymbol{A}_{k j}$. The Rsd, Rdd, and Rud of $x_{i j}^{k}$ are defined as

$$
\begin{aligned}
\xi_{i j}^{k} & =\frac{d\left(x_{i j}^{k}, A_{j}^{\min }\right)}{d\left(x_{i j}^{k}, A_{j}^{\max }\right)+d\left(x_{i j}^{k}, A_{j}^{\min }\right)}, \\
\zeta_{i j}^{k} & =\frac{d\left(x_{i j}^{k}, A_{j}^{\max }\right)}{d\left(x_{i j}^{k}, A_{j}^{\max }\right)+d\left(x_{i j}^{k}, A_{j}^{\min }\right)}, \\
\eta_{i j}^{k} & =\min \left\{\frac{d\left(x_{i j}^{k}, A_{j}^{\min }\right)}{d\left(x_{i j}^{k}, A_{j}^{\max }\right)}, \frac{d\left(x_{i j}^{k}, A_{j}^{\max }\right)}{d\left(x_{i j}^{k}, A_{j}^{\min }\right)}\right\},
\end{aligned}
$$

respectively, where $\xi_{i j}^{k}$, $\zeta_{i j}^{k}$, and $\eta_{i j}^{k}$ denote the Rsd, Rdd, and Rud given by $d_{i}$ on attribute $A_{j}$ in the alternative $S_{k}$, $d\left(x_{i j}^{k}, A_{j}^{\max }\right)$ is the distance between $x_{i j}^{k}$ and the largest grade $A_{j}^{\max }$ of the attribute $A_{j}$, and $d\left(x_{i j}^{k}, A_{j}^{\min }\right)$ is the distance between $x_{i j}^{k}$ and the smallest grade $A_{j}^{\min }$ of the attribute $A_{j}$.

For Example 1. Consider that $x_{11}^{1}=(4.6,7.2,8.3)$ is a TFN in the ten-mark system; then $A_{j}^{\min }=(0,0,0), A_{j}^{\max }=$ $(10,10,10)$. According to (19)-(21), the Rsd, Rdd, and Rud of $x_{11}^{1}$ are calculated, respectively, as $\xi_{11}^{1}=0.67, \zeta_{11}^{1}=0.33$, and $\eta_{11}^{1}=0.49$.

Theorem 8. Rud $\eta_{i j}^{k}$ of $x_{i j}^{k}$ in $\boldsymbol{A}_{k j}$ has the following properties.

(EPI) If $x_{i j}^{k}=A_{j}^{\max }$ or $x_{i j}^{k}=A_{j}^{\min }$, then $\eta_{i j}^{k}=0$, which means that $A_{j}^{\min }$ and $A_{j}^{\max }$ are not fuzzy since $A_{j}^{\min }$ and $A_{j}^{\max }$ are crisp sets.

(EP2) If $d\left(x_{i j}^{k}, A_{j}^{\max }\right)=d\left(x_{i j}^{k}, A_{j}^{\min }\right)$ (namely, $x_{i j}^{k}$ is the middle point), then $\eta_{i j}^{k}=1$, which means that $x_{i j}^{k}$ is the fuzziest element.
(EP3) $\eta_{i j}^{k} \leq \eta_{t j}^{k}(i, t \in M=\{1,2, \ldots, m\})$, if $\eta_{i j}^{k}$ is less fuzzy than $\eta_{i j}^{k}$, i.e.,

$$
\begin{aligned}
& \frac{d\left(x_{i j}^{k}, A_{j}^{\min }\right)}{d\left(x_{i j}^{k}, A_{j}^{\max }\right)} \leq \frac{d\left(x_{t j}^{k}, A_{j}^{\min }\right)}{d\left(x_{t j}^{k}, A_{j}^{\max }\right)}, \\
& \text { for } d\left(x_{i j}^{k}, A_{j}^{\min }\right) \leq d\left(x_{i j}^{k}, A_{j}^{\max }\right), d\left(x_{t j}^{k}, A_{j}^{\min }\right) \leq d\left(x_{t j}^{k}, A_{j}^{\max }\right) ; \\
& \frac{d\left(x_{i j}^{k}, A_{j}^{\max }\right)}{d\left(x_{i j}^{k}, A_{j}^{\min }\right)} \leq \frac{d\left(x_{t j}^{k}, A_{j}^{\min }\right)}{d\left(x_{t j}^{k}, A_{j}^{\max }\right)}, \\
& \text { for } d\left(x_{i j}^{k}, A_{j}^{\min }\right) \geq d\left(x_{i j}^{k}, A_{j}^{\max }\right), d\left(x_{t j}^{k}, A_{j}^{\min }\right) \leq d\left(x_{t j}^{k}, A_{j}^{\max }\right) ; \\
& \frac{d\left(x_{i j}^{k}, A_{j}^{\min }\right)}{d\left(x_{i j}^{k}, A_{j}^{\max }\right)} \leq \frac{d\left(x_{t j}^{k}, A_{j}^{\max }\right)}{d\left(x_{t j}^{k}, A_{j}^{\min }\right)}, \\
& \text { for } d\left(x_{i j}^{k}, A_{j}^{\min }\right) \leq d\left(x_{i j}^{k}, A_{j}^{\max }\right), d\left(x_{t j}^{k}, A_{j}^{\min }\right) \geq d\left(x_{t j}^{k}, A_{j}^{\max }\right) ; \\
& \frac{d\left(x_{i j}^{k}, A_{j}^{\max }\right)}{d\left(x_{i j}^{k}, A_{j}^{\min }\right)} \leq \frac{d\left(x_{t j}^{k}, A_{j}^{\max }\right)}{d\left(x_{t j}^{k}, A_{j}^{\min }\right)}, \\
& \text { for } d\left(x_{i j}^{k}, A_{j}^{\min }\right) \geq d\left(x_{i j}^{k}, A_{j}^{\max }\right), d\left(x_{t j}^{k}, A_{j}^{\min }\right) \geq d\left(x_{t j}^{k}, A_{j}^{\max }\right) .
\end{aligned}
$$

It is easy to prove that Rud $\eta_{i j}^{k}$ meets the properties (EP1)(EP3), which are consistent with the axioms (1)-(3) of fuzzy entropy based on distance in [48]. From a geometric standpoint, $A_{j}^{\max }$ and $A_{j}^{\min }$ in the rating system are nonfuzzy which can correspond to the position $M$ and position $N$ (Figure 2). When a fuzzy number $x_{i j}^{k}$ is moved from position $M($ or $N$ ) towards middle position $O$, the distance from $x_{i j}^{k}$ to $M$ is close to $N$. Meanwhile, $x_{i j}^{k}$ become more and more vague; i.e., $\eta_{i j}^{k}$ is getting bigger and bigger. Particularly, when $x_{i j}^{k}$ is in position $P$, the distance from $x_{i j}^{k}$ to $M$ is equal to $N$. So, the fuzzy number $x_{i j}^{k}$ is the fuzziest, i.e., $\eta_{i j}^{k}=1$. According to the analysis above, it is reasonable that Rud measures the uncertainty of the original assessment. It is worth mentioning that (21) is suitable for different forms of decision data such as INs, TFNs, TIFNs, and TrIFNs.

Remark 9. From (19)-(21), the extraction method in this paper relies on just the largest grade $A_{j}^{\max }$ and the smallest grade $A_{j}^{\min }$ of the attribute $A_{j}$, while the methods $[35,37]$ rely on $A_{j}^{\max }$ and $A_{j}^{\min }$ as well as the middle grade $A_{j}^{\mathrm{mid}}$ of the attribute $A_{j}$. However, it is hard to determine the middle grade for some sets of TIFNs [43] and TrIFNs [49, 50]. Hence, the proposed extraction method is more effective and simple. 
Remark 10. When $\boldsymbol{A}_{k j}$ is a cost attribute vector, the Rud of $x_{i j}^{k}$ can be derived by (21). The Rsd and Rdd of $x_{i j}^{k}$ can be rewritten as

$$
\begin{aligned}
\xi_{i j}^{k} & =\frac{d\left(x_{i j}^{k}, A_{j}^{\max }\right)}{d\left(x_{i j}^{k}, A_{j}^{\max }\right)+d\left(x_{i j}^{k}, A_{j}^{\min }\right)}, \\
\zeta_{i j}^{k} & =\frac{d\left(x_{i j}^{k}, A_{j}^{\min }\right)}{d\left(x_{i j}^{k}, A_{j}^{\max }\right)+d\left(x_{i j}^{k}, A_{j}^{\min }\right)}
\end{aligned}
$$

Remark 11. For the benefit attribute vector $\boldsymbol{A}_{k j}=\left(x_{1 j}^{k}\right.$, $\left.x_{2 j}^{k}, \ldots, x_{m j}^{k}\right), \xi_{i j}^{k}, \zeta_{i j}^{k}$, and $\eta_{i j}^{k}$ of each element are composed of support set $\xi_{k j}$, opposition set $\zeta_{k j}$, and uncertain set $\eta_{k j}$ of $\boldsymbol{A}_{k j}$ which can be defined as $\xi_{k j}=\left\{\xi_{1 j}^{k}, \xi_{2 j}^{k}, \ldots, \xi_{m j}^{k}\right\}$ and $\zeta_{k j}=$ $\left\{\zeta_{1 j}^{k}, \zeta_{2 j}^{k}, \ldots, \zeta_{m j}^{k}\right\}$ and $\eta_{k j}=\left\{\eta_{1 j}^{k}, \eta_{2 j}^{k}, \ldots, \eta_{m j}^{k}\right\}$, respectively.

3.2.2. Calculate the Mode. For the support set $\xi_{k j}=\left\{\xi_{1 j}^{k}\right.$, $\left.\xi_{2 j}^{k}, \ldots, \xi_{m j}^{k}\right\}$, the mode of the TFN is located in the center around which the Rsd $\xi_{i j}^{k}(i=1,2, \ldots, m)$ gather. Inspired by the literature $[49,50]$, the more consistent it is with the rest of $\xi_{k j}$, the greater the importance of the Rsd $\xi_{i j}^{k}$ given by DM $D_{i}$. That is to say, the weighted average of the collection $\xi_{k j}$ can be regarded as its mode. Here, we utilize the distance between $\xi_{i j}^{k}$ and $\xi_{e j}^{k}$ to define the consistency degree of $D_{i}$ on support set $\xi_{k j}$ to the rest of experts, which can be obtained by

$$
C I_{i j}^{k}=\frac{1}{m-1} \sum_{i=1, i \neq j}^{m}\left(1-d\left(\xi_{i j}^{k}, \xi_{e j}^{k}\right)\right),
$$

where $d(\cdot)$ is the distance between $\xi_{i j}^{k}$ and other Rsds in $\xi_{k j}$. Clearly, $0 \leq S_{k} \leq 1$.

Generally, an expert's Rsd is more important if he/she is more similar to the group's Rsd. In other words, the larger the value of $C I_{i j}^{k}$ is, the more important $\xi_{k j}$ is. Thus, the weight of $D_{i}$ on $\xi_{k j}$ can be obtained by

$$
u_{i j}^{k}=\frac{C I_{i j}^{k}}{\sum_{i=1}^{m} C I_{i j}^{k}} \quad(i=1,2, \ldots, m) .
$$

Then the mode of $\delta_{k j}$ for support set $\xi_{k j}$ is derived as

$$
m\left(\xi_{k j}\right)=\sum_{i=1}^{m} u_{i j}^{k} \xi_{i j}^{k}
$$

Similarly, we have the mode $m\left(\zeta_{k j}\right)$ of opposition set $\zeta_{k j}$.

3.2.3. Construct Qst and Qdt. Note that the membership degree and nonmembership degree of a TIFN are TFNs rather than real numbers. Moreover, $\xi_{i j}^{k}$ and $\zeta_{i j}^{k}$ are real numbers which are difficult to express the imprecise and vague experts' subjective judgment. By doing this, the TFNs of $\xi_{i j}^{k}$ and $\zeta_{i j}^{k}$ are commonly used to represent Qsd and Qdd of $\boldsymbol{A}_{k j}$ since TFN is characterized by a membership function. Thus, it is necessary to construct the Qst and Qdt of $\boldsymbol{A}_{k j}$. As per the definition of TFN, the corresponding TFNs of $\xi_{k j}$ and $\zeta_{k j}$ can be constructed as follows.

Definition 12. For the attribute vector $\boldsymbol{A}_{k j}$, the Qst $\delta_{k j}$ and Qdt $\gamma_{k j}$ of alternative $S_{k}$ on attribute $A_{j}$ are defined as

$$
\begin{array}{ll}
\delta_{k j}=\left(\min \left\{\xi_{k j}\right\}, m\left(\xi_{k j}\right), \max \left\{\xi_{k j}\right\}\right), & \\
& (k \in P, j \in N) \\
\gamma_{k j}=\left(\min \left\{\zeta_{k j}\right\}, m\left(\zeta_{k j}\right), \max \left\{\zeta_{k j}\right\}\right), & \\
& (k \in P, j \in N)
\end{array}
$$

where the $\min \left\{\xi_{k j}\right\}$ and $\max \left\{\xi_{k j}\right\}$ are the minimum value and maximum value of the support set $\xi_{k j}$ and $\min \left\{\zeta_{k j}\right\}$ and $\max \left\{\zeta_{k j}\right\}$ are the minimum value and maximum value of opposition set $\zeta_{k j}$. For the convenience of discussion, the pair $\left(\delta_{k j}, \gamma_{k j}\right)$ is called a quasi-ITFN.

Remark 13. To calculate the mode of triangular fuzzy numbers $\delta_{k j}$ and $\gamma_{k j}$, this paper employs the weighted averaging value that considers the distribution of ratings, whereas some works used the mean value method. The essential difference is that the current method takes the consistency of the group into account, while the mean value method is based on statistical assumptions.

3.2.4. Inducing an ITFN. Finally, an ITFN is induced from the Qst and Qdt of alternative $S_{k}$ on the attribute $A_{j}$ by the following normalized method. Let $\alpha_{k j}=$ $\left(\left(t_{k j}^{l}, t_{k j}^{m}, t_{k j}^{h}\right),\left(f_{k j}^{l}, f_{k j}^{m}, f_{k j}^{h}\right)\right)(k \in P, j \in N)$ be the induced ITFN by the attribute vector $\boldsymbol{A}_{k j}$, and $\operatorname{mean}\left(\eta_{k j}\right)=$ $(1 / m) \sum_{i=1}^{m} \eta_{i j}^{k}$ is the uncertain degree of $\boldsymbol{A}_{k j}$, To satisfy the conditions in (3) and consider the influence of uncertain degree, the values of $t_{k j}^{l}, t_{k j}^{m}, t_{k j}^{h}, f_{k j}^{l}, f_{k j}^{m}$, and $f_{k j}^{h}$ can be computed as follows:

$$
\begin{aligned}
t_{k j}^{l} & =\frac{\min \left\{\xi_{k j}\right\}}{\psi_{k j}}, \\
t_{k j}^{m} & =\frac{\operatorname{mean}\left(\xi_{k j}\right)}{\psi_{k j}}, \\
t_{k j}^{h} & =\frac{\max \left\{\xi_{k j}\right\}}{\psi_{k j}}, \\
f_{k j}^{l} & =\frac{\min \left\{\zeta_{k j}\right\}}{\psi_{k j}}, \\
f_{k j}^{m} & =\frac{\operatorname{mean}\left(\zeta_{k j}\right)}{\psi_{k j}},
\end{aligned}
$$




$$
f_{k j}^{h}=\frac{\max \left\{\zeta_{k j}\right\}}{\psi_{k j}}, \begin{aligned}
& \\
& (k \in P, j \in N)
\end{aligned}
$$

respectively, where $\psi_{k j}=(1 / 5)\left(\min \left\{\xi_{k j}\right\}+m\left(\xi_{k j}\right)+\min \left\{\zeta_{k j}\right\}+\right.$ $\left.m\left(\zeta_{k j}\right)+\operatorname{mean}\left(\eta_{k j}\right)\right)+\max \left\{\xi_{k j}\right\}+\max \left\{\zeta_{k j}\right\}$.

Apparently, $t_{k j}^{l}, t_{k j}^{m}, t_{k j}^{h}, f_{k j}^{l}, f_{k j}^{m}$, and $f_{k j}^{h}$ satisfy (3). Thus, $\alpha_{k j}=\left(\left(t_{k j}^{l}, t_{k j}^{m}, t_{k j}^{h}\right),\left(f_{k j}^{l}, f_{k j}^{m}, f_{k j}^{h}\right)\right)$ is an ITFN. Namely, all the attribute values in the vector $\boldsymbol{A}_{k j}$ can be aggregated into an ITFN $\alpha_{k j}$.

For Example 2. Consider that $\boldsymbol{A}_{k j}=((4.6,7.2,8.3)$, $(3.4,4.6,6.7),(5.6,6.2,8.2),(4.5,6.5,10),(3.7,6.5,7.2))$ is a TFN vector in the ten-mark system; then $A_{j}^{\min }=(0,0,0)$,
$A_{j}^{\max }=(10,10,10)$. According to (19)-(21), the support set, opposition set, and uncertain set of $\boldsymbol{A}_{k j}$ are calculated, respectively, as $\xi_{k j}=\{0.67,0.49,0.70,0.65,0.58\}, \zeta_{k j}=\{0.33$, $0.51,0.30,0.35,0.42\}$, and $\eta_{k j}=\{0.49,0.39,0.52,0.5,0.44\}$. Subsequently, the mode of the sets $\xi_{k j}$ and $\zeta_{k j}$ is calculated from (25)-(27) to be $m\left(\xi_{k j}\right)=0.62$ and $m\left(\zeta_{k j}\right)=0.38$. It follows from (28) and (29) that $\delta_{k j}=(0.49,0.62,0.70)$ and $\gamma_{k j}=(0.30,0.38,0.51)$. Finally, by using (29), the induced ITFN associated with $\boldsymbol{A}_{k j}$ is derived to be $\alpha_{k j}=$ $((0.349,0.445,0.499),(0.214,0.268,0.364))$.

\section{A Novel Approach for Heterogeneous MAGDM Problems}

According to the proposed aggregation method, the collective decision matrix $\boldsymbol{X}=\left(r_{k j}\right)_{p \times n}$ is aggregated as follows:

$$
\left.\begin{array}{ccccc} 
& A_{1} & A_{2} & \cdots & A_{n} \\
S_{1} & \left(\left(\left(t_{11}^{l}, t_{11}^{m}, t_{11}^{h}\right),\left(f_{11}^{l}, f_{11}^{m}, f_{11}^{h}\right)\right)\right. & \left(\left(t_{12}^{l}, t_{12}^{m}, t_{12}^{h}\right),\left(f_{12}^{l}, f_{12}^{m}, f_{12}^{h}\right)\right) & \cdots & \left(\left(t_{1 n}^{l}, t_{1 n}^{m}, t_{1 n}^{h}\right),\left(f_{1 n}^{l}, f_{1 n}^{m}, f_{1 n}^{h}\right)\right) \\
S_{2} & \left(\left(t_{21}^{l}, t_{21}^{m}, t_{21}^{h}\right),\left(f_{21}^{l}, f_{21}^{m}, f_{21}^{h}\right)\right) & \left(\left(t_{22}^{l}, t_{22}^{m}, t_{22}^{h}\right),\left(f_{22}^{l}, f_{22}^{m}, f_{22}^{h}\right)\right) & \cdots & \left(\left(t_{2 n}^{l}, t_{2 n}^{m}, t_{2 n}^{h}\right),\left(f_{2 n}^{l}, f_{2 n}^{m}, f_{2 n}^{h}\right)\right) \\
\vdots & \vdots & \vdots & \vdots & \vdots \\
S_{p} & \left(\left(t_{p 1}^{l}, t_{p 1}^{m}, t_{p 1}^{h}\right),\left(f_{p 1}^{l}, f_{p 1}^{m}, f_{p 1}^{h}\right)\right) & \left(\left(t_{p 2}^{l}, t_{p 2}^{m}, t_{p 2}^{h}\right),\left(f_{p 2}^{l}, f_{p 2}^{m}, f_{p 2}^{h}\right)\right) & \cdots & \left(\left(t_{p n}^{l}, t_{p n}^{m}, t_{p n}^{h}\right),\left(f_{p n}^{l}, f_{p n}^{m}, f_{p n}^{h}\right)\right)
\end{array}\right)
$$

where $r_{k j}=\left(\left(t_{k j}^{l}, t_{k j}^{m}, t_{k j}^{h}\right),\left(f_{k j}^{l}, f_{k j}^{m}, f_{k j}^{h}\right)\right)(k \in P, j \in N)$ are ITFNs aggregated by the attribute vector $\boldsymbol{A}_{k j}$ in $\boldsymbol{X}^{k}$.

Assume that the weight vector $\boldsymbol{w}=\left(w_{1}, w_{2}, \ldots, w_{n}\right)$ of attributes is fully known; by (4), we can easily obtain the comprehensive rating $r_{k}=\left(\left(t_{k}^{l}, t_{k}^{m}, t_{k}^{h}\right),\left(f_{k}^{l}, f_{k}^{m}, f_{k}^{h}\right)\right)$ of the alternative $S_{k}$. When $\boldsymbol{w}$ is incompletely known, we may utilize the following programming model to establish the attribute weights.

4.1. Determination of Attribute Weights. As all known, it is reasonable to determine the attribute weights by making alternative similar to the positive ideal solution (PIS) and at the same time far away from the negative ideal solution (NIS) as far as possible. Let $S^{+}=\left\{r_{1}^{+}, r_{2}^{+}, \ldots, r_{n}^{+}\right\}$and $S^{-}=$ $\left\{r_{1}^{-}, r_{2}^{-}, \ldots, r_{n}^{-}\right\}$be the intuitionistic triangular fuzzy PIS and NIS, respectively, where

$$
\begin{aligned}
r_{j}^{+} & =\left(\left(\max _{k} t_{k j}^{l}, \max _{k} t_{k j}^{m}, \max _{k} t_{k j}^{h}\right),\right. \\
& \left(\min _{k} f_{k j}^{l}, \min _{k} f_{k j}^{m}, \min _{k} f_{k j}^{h}\right) \\
& =\left(\left(t_{j}^{l^{+}}, t_{j}^{m^{+}}, t_{j}^{h^{+}}\right),\left(f_{j}^{l^{+}}, f_{j}^{m^{+}}, f_{j}^{h^{+}}\right)\right), \\
r_{j}^{-} & =\left(\left(\min _{k} t_{k j}^{l}, \min _{k} t_{k j}^{m}, \min _{k} t_{k j}^{h}\right),\right. \\
& \left(\max _{k} f_{k j}^{l}, \max _{k} f_{k j}^{m}, \max _{k} f_{k j}^{h}\right) \\
& =\left(\left(t_{j}^{l^{-}}, t_{j}^{m-}, t_{j}^{h^{-}}\right),\left(f_{j}^{l^{-}}, f_{j}^{m-}, f_{j}^{h^{-}}\right)\right) .
\end{aligned}
$$

$(j \in N=\{1,2, \ldots, n\})$. Based on (5), we can define the similarity degree between alternative $S_{k}(k \in P=\{1,2, \ldots, p\})$ and intuitionistic triangular fuzzy ideal solution, shown as follows:

$$
\begin{aligned}
\vartheta_{k}^{+} & =1-\sum_{j=1}^{p} w_{j} *\left[\frac { 1 } { 1 2 } \left(\left|t_{k j}^{l}-t_{j}^{l^{+}}\right|+\left|t_{k j}^{m}-t_{j}^{m+}\right|+\mid t_{k j}^{h}\right.\right. \\
& \left.-t_{j}^{h^{+}}|+| f_{k j}^{l}-f_{j}^{l^{+}}|+| f_{k j}^{m}-f_{j}^{m^{+}}|+| f_{k j}^{h}-f_{j}^{h^{+}} \mid\right) \\
& +\frac{1}{2} \max \left(\left|t_{k j}^{l}-t_{j}^{l^{+}}\right|,\left|t_{k j}^{m}-t_{j}^{m+}\right|,\left|t_{k j}^{h}-t_{j}^{h^{+}}\right|,\right. \\
& \left.\left.\left|f_{k j}^{l}-f_{j}^{l^{+}}\right|,\left|f_{k j}^{m}-f_{j}^{m+}\right|,\left|f_{k j}^{h}-f_{j}^{h^{+}}\right|\right)\right] \\
\vartheta_{k}^{-} & =1-\sum_{j=1}^{p} w_{j} *\left[\frac { 1 } { 1 2 } \left(\left|t_{k j}^{l}-t_{j}^{l^{-}}\right|+\left|t_{k j}^{m}-t_{j}^{m-}\right|+\mid t_{k j}^{h}\right.\right. \\
& \left.-t_{j}^{h^{-}}|+| f_{k j}^{l}-f_{j}^{l^{-}}|+| f_{k j}^{m}-f_{j}^{m^{-}}|+| f_{k j}^{h}-f_{j}^{h^{-}} \mid\right) \\
& +\frac{1}{2} \max \left(\left|t_{k j}^{l}-t_{j}^{l^{-}}\right|,\left|t_{k j}^{m}-t_{j}^{m-}\right|,\left|t_{k j}^{h}-t_{j}^{h^{-}}\right|,\right. \\
& \left.\left.\left|f_{k j}^{l}-f_{j}^{l^{-}}\right|,\left|f_{k j}^{m}-f_{j}^{m-}\right|,\left|f_{k j}^{h}-f_{j}^{h^{-}}\right|\right)\right]
\end{aligned}
$$

Then, a multiple objective linear mathematical programming model is constructed as follows:

$$
\begin{array}{ll}
\max & \left\{\vartheta_{k}^{+}-\vartheta_{k}^{-}\right\} \quad(k \in P) \\
\text { s.t. } & w \in \Omega
\end{array}
$$


For a multiple objective programming, there are several solution methods. Here, we apply the Max-Min method. Let $\rho=\min \left\{\vartheta_{k}^{+}-\vartheta_{k}^{-}\right\}$. By using the Max-Min method, (36) can be solved by the following single objective linear programming model:

$$
\begin{array}{ll}
\max & \rho \\
\text { s.t. } & \left\{\begin{array}{l}
\vartheta_{k}^{+}-\vartheta_{k}^{-} \geq \rho \quad(k \in P) \\
\boldsymbol{w} \in \Omega
\end{array}\right.
\end{array}
$$

By plugging (34) and (35) in (37), it is easily seen that we can derive the attribute weights since the optimal solution of (37) is a Pareto optimal solution of (36).

Remark 14. To obtain the weights of attributes in the intuitionistic triangular fuzzy environment, the current methodology first combines a multiple objective mathematical programming model with TOPSIS idea based on the above collective decision matrix. Then, this model can be solved by Max-Min method, which is relatively simple. However, Li and Chen [49] determined the attribute weights by expected weight value that involved options of decision makers. Shan and $\mathrm{Xu}$ [42] gave the attribute weights in advance. Therefore, our method could be more reasonable and objective.

Thus, the ranking order of the alternative $S_{k}$ can be conducted by the following relative closeness coefficient (RCC):

$$
R C C_{k}=\frac{\vartheta_{k}^{+}}{\vartheta_{k}^{+}+\vartheta_{k}^{-}}
$$

where $0 \leq R C C_{k} \leq 1,(k \in P)$. It is obvious that the larger $R C C_{k}$, the better the alternative $S_{k}$.

4.2. Procedure of the Proposed Aggregation Method for Heterogeneous MAGDM. On the basis of the above analysis, a new approach to heterogeneous MAGDM problems involves the following primary steps.

Step 1. Obtain the decision matrix of alternative $S_{k}(k=$ $1,2, \ldots, p)$ taking the form of $\boldsymbol{Y}^{k}=\left(y_{i j}^{k}\right)_{m \times n}$, by (18).

Step 2. Convert the decision matrices $\boldsymbol{X}^{k}=\left(x_{i j}^{k}\right)_{m \times n}(k=$ $1,2, \ldots, p)$ into a collective decision matrix $\boldsymbol{X}=\left(r_{k j}\right)_{p \times n}$ through the aggregation method developed in Section 3. Based on each column vector $\boldsymbol{A}_{k j}$ in the matrix $\boldsymbol{X}^{k}$, conduct the following substeps.

Step 2.1. Compute the Rsd, Rdd, and Rud of $x_{i j}^{k}$ by plugging the Hamming distance of different attribute types into (19), (20), (21), (23), and (24), and construct support set $\xi_{k j}$, opposition set $\zeta_{k j}$, and uncertain set $\eta_{k j}$ of $\boldsymbol{A}_{k j}$.

Step 2.2. Calculate the mode of the support set $\xi_{k j}$ and opposition set $\zeta_{k j}$ of $\boldsymbol{A}_{k j}$ by (25)-(27).
Step 2.3. Construct the Qst $\delta_{k j}$ and Qdt $\gamma_{k j}$ of $\boldsymbol{A}_{k j}$ by (28) and (29).

Step 2.4. Induce the corresponding ITFN of $\boldsymbol{A}_{k j}$ using (30).

Step 3. Determine the attribute weights by constructing a multiple objective programming model. The detailed steps are as follows.

Step 3.1. Define the intuitionistic triangular fuzzy PIS $S^{+}=$ $\left\{r_{1}^{+}, r_{2}^{+}, \ldots, r_{n}^{+}\right\}$and NIS $S^{-}=\left\{r_{1}^{-}, r_{2}^{-}, \ldots, r_{n}^{-}\right\}$by (32) and (33), respectively.

Step 3.2. Compute the similarity degrees $\vartheta_{k}^{+}$and $\vartheta_{k}^{-}$from the elements at the $k$ th row of the collective decision matrix $\boldsymbol{X}=$ $\left(r_{k j}\right)_{p \times n}$ to PIS $S^{+}$and NIS $S^{-}$by (34) and (35), respectively.

Step 3.3. Construct a multiple objective programming model based on (36).

Step 3.4. Convert the above model into a single objective programming model by (37).

Step 3.5. Obtain the optimal weights of attributes by solving the linear programming model.

Step 4. Calculate the similarity degrees $\vartheta_{k}^{+}$and $\vartheta_{k}^{-}$of alternative $S_{k}(k \in P)$ by the obtained attribute weights and (34) and (35).

Step 5. Calculate the RCC of alternative $S_{k}(k \in P)$ by (38).

Step 6. Rank the alternatives according to the RCC and select the best one.

The decision procedure of the proposed algorithm may be depicted in Figure 3.

\section{A Trustworthy Seller Selection Problem and Comparison Analyses}

To demonstrate the efficacy of the proposed HMAGDM method, this section gives a trustworthy seller selection example and conducts comparison analyses with the ones of the existing methods $[34,35,37]$.

5.1. A Trustworthy Seller Selection Example and Its Solution Procedure. Online service trading usually takes place between parties who are autonomous, in an environment where the buyer often has not enough information about the seller and goods. Many scholars think that trust is a prerequisite for successful trading. Therefore, it is very important that buyers can identify the most trustworthy seller. Suppose that a consumer desires to select a trustworthy seller. After preliminary screening, four candidate sellers $S_{1}, S_{2}, S_{3}$, and $S_{4}$ remain to be further evaluated. Based on detailed seller ratings, the decision-making committee assesses the 


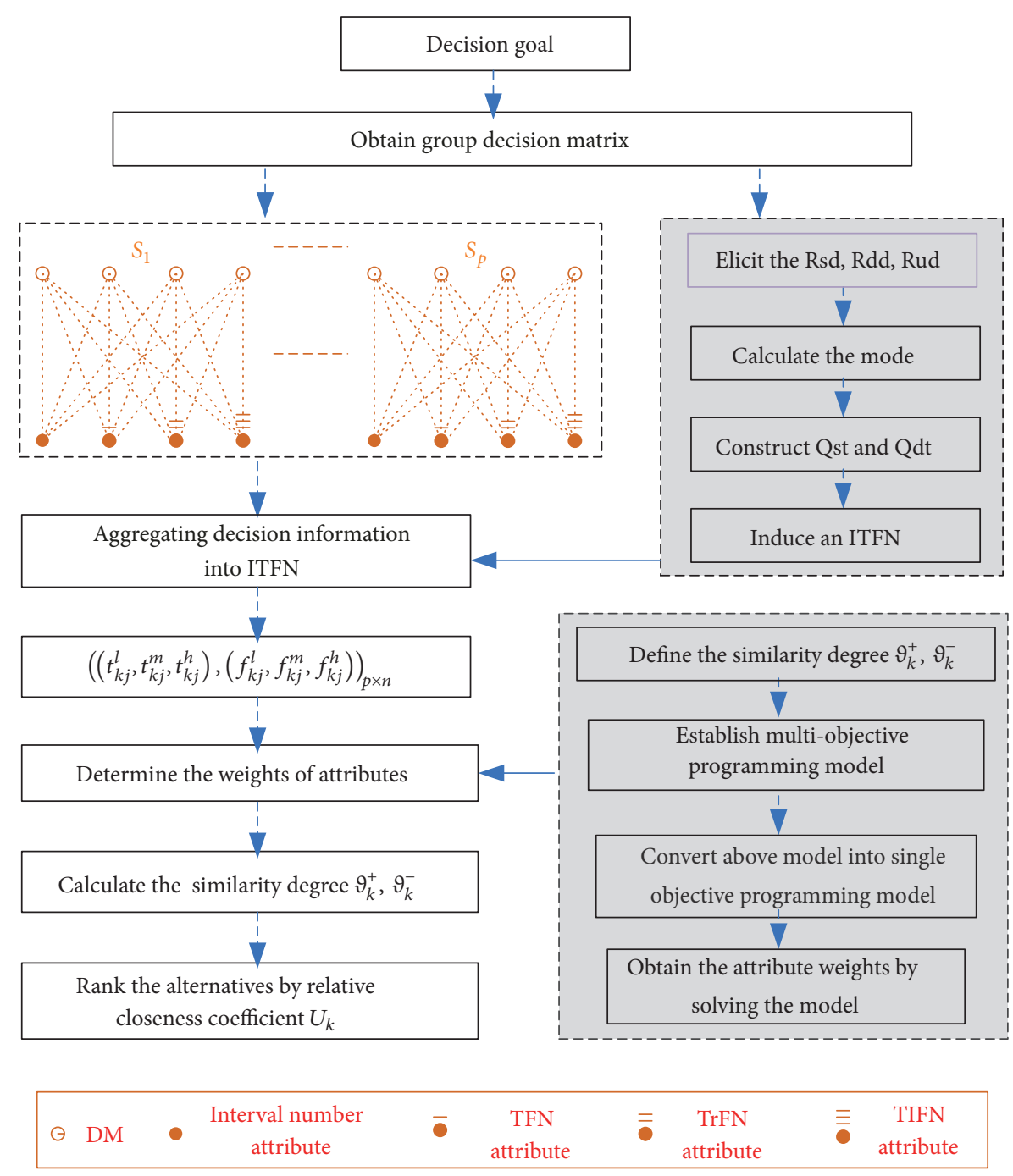

FIGURE 3: The framework of the proposed heterogeneous MAGDM method.

four candidate sellers according to the five trust factors, including product quality $\left(A_{1}\right)$, service attitude $\left(A_{2}\right)$, website usability $\left(A_{3}\right)$, response time $\left(A_{4}\right)$, and shipping speed $\left(A_{5}\right)$.

Product quality $\left(A_{1}\right)$, service attitude $\left(A_{2}\right)$, and website usability $\left(A_{3}\right)$ given by DMs with a one-mark system are all benefit attributes. It is better to use TFNs to assess product quality $\left(A_{1}\right)$. For service attitude $\left(A_{2}\right)$, the experts like to provide the lower and upper limits and the most possible intervals; thus the assessments of $A_{2}$ can be represented by
TrFNs. The website usability $\left(A_{3}\right)$ is expressed by TIFNs, while response time $\left(A_{4}\right)$ and shipping speed $\left(A_{5}\right)$ given by DMs with a ten-mark system both are cost attributes. The assessments of the sellers on $A_{4}$ can be represented by RNs. Due to the uncertainty of shipping speed, INs are suitably utilized to represent the assessments of shipping speed $\left(A_{5}\right)$. The assessments of four sellers on five attributes given by five experts are listed in Table 1. The attributes' importance is incomplete and experts give incomplete information on the attributes' importance as follows:

$$
\Omega=\left\{\begin{array}{l}
w_{1} \geq 0.1 ; w_{2}-w_{1} \leq 0.05 ; w_{3}-w_{1} \leq 0.05 ; w_{5}-w_{1} \leq 0.05 ; \\
w_{1}+w_{3}+w_{5} \leq 0.6 ; w_{4} \leq 0.2 ; w_{1}+w_{2}+w_{3}+w_{4}+w_{5}=1 .
\end{array}\right\}
$$

Obviously, the decision problem mentioned above is a heterogeneous MAGDM problem involving five different formats of data: RNs, INs, TFNs, TrFNs, and TIFNs.
To address this problem, we apply the proposed decision method to the selection of the trustworthy sellers below. 
TABLE 1: The decision matrix of four alternatives.

\begin{tabular}{|c|c|c|c|c|c|c|}
\hline Sellers & DMs & $A_{1}$ & $A_{2}$ & $A_{3}$ & $A_{4}$ & $A_{5}$ \\
\hline \multirow[t]{5}{*}{$S_{1}$} & $D_{1}$ & $(0.46,0.72,0.83)$ & $(0.2,0.3,0.5,1.0)$ & $((0.33,0.5,0.67), 0.5,0.3)$ & 3.4 & {$[2,6]$} \\
\hline & $D_{2}$ & $(0.34,0.46,0.67)$ & $(0.4,0.5,0.6,0.8)$ & $((0.33,0.5,0.67), 0.4,0.5)$ & 5.4 & {$[1,3]$} \\
\hline & D3 & $(0.56,0.62,0.82)$ & $(0.4,0.4,0.5,0.8)$ & $((0.33,0.5,0.67), 0.6,0.1)$ & 7.2 & {$[1,6]$} \\
\hline & $D_{4}$ & $(0.45,0.65,1.00)$ & $(0.2,0.3,0.5,0.9)$ & $((0.5,0.67,0.83), 0.7,0.2)$ & 8.1 & {$[1,4]$} \\
\hline & $D_{5}$ & $(0.37,0.65,0.72)$ & $(0.0,0.1,0.3,0.7)$ & $((0.17,0.33,0.5), 0.4,0.6)$ & 5.9 & {$[3,8]$} \\
\hline \multirow[t]{5}{*}{$S_{2}$} & $D_{1}$ & $(0.51,0.67,0.73)$ & $(0.2,0.3,0.4,0.7)$ & $((0.67,0.83,1.0), 0.6,0.3)$ & 4.2 & {$[3,6]$} \\
\hline & $\mathrm{D}_{2}$ & $(0.35,0.64,0.73)$ & $(0.3,0.4,0.4,0.9)$ & $((0.33,0.5,0.67), 0.4,0.5)$ & 5.4 & {$[2,5]$} \\
\hline & D3 & $(0.66,0.82,1.00)$ & $(0.2,0.2,0.4,0.5)$ & $((0.67,0.83,1.0), 0.6,0.2)$ & 7.2 & {$[4,7]$} \\
\hline & $D_{4}$ & $(0.34,0.51,0.77)$ & $(0.5,0.6,0.8,0.8)$ & $((0.17,0.33,0.5), 0.7,0.2)$ & 10.0 & {$[1,3]$} \\
\hline & $D_{5}$ & $(0.68,0.82,1.00)$ & $(0.1,0.1,0.2,0.5)$ & $((0.67,0.83,1.0), 0.3,0.6)$ & 7.1 & {$[6,7]$} \\
\hline \multirow[t]{5}{*}{$S_{3}$} & $D_{1}$ & $(0.52,0.66,0.81)$ & $(0.5,0.6,0.6,0.8)$ & $((0.0,0.17,0.33), 0.5,0.3)$ & 7.8 & {$[2,4]$} \\
\hline & $\mathrm{D}_{2}$ & $(0.39,0.66,0.68)$ & $(0.4,0.5,0.7,0.8)$ & $((0.33,0.5,0.67), 0.4,0.5)$ & 6.5 & {$[2,4]$} \\
\hline & D3 & $(0.62,0.67,0.84)$ & $(0.3,0.3,0.5,0.9)$ & $((0.0,0.17,0.33), 0.6,0.1)$ & 4.3 & {$[1,3]$} \\
\hline & $D_{4}$ & $(0.58,0.74,1.00)$ & $(0.4,0.4,0.6,0.8)$ & $((0.33,0.5,0.67), 0.6,0.3)$ & 8.3 & {$[3,5]$} \\
\hline & $D_{5}$ & $(0.44,0.50,0.67)$ & $(0.3,0.3,0.4,0.5)$ & $((0.5,0.67,0.83), 0.4,0.6)$ & 5.5 & {$[4,7]$} \\
\hline \multirow[t]{5}{*}{$S_{4}$} & $D_{1}$ & $(0.34,0.47,0.67)$ & $(0.2,0.3,0.4,0.5)$ & $((0.5,0.67,0.83), 0.4,0.4)$ & 4.9 & {$[6,7]$} \\
\hline & $\mathrm{D}_{2}$ & $(0.59,0.66,0.68)$ & $(0.2,0.3,0.4,0.4)$ & $((0.5,0.67,0.83) 0.6,0.4)$ & 8.1 & {$[5,6]$} \\
\hline & D3 & $(0.47,0.56,0.67)$ & $(0.1,0.1,0.2,0.7)$ & $((0.33,0.5,0.67), 0.6,0.1)$ & 3.9 & {$[1,10]$} \\
\hline & $D_{4}$ & $(0.45,0.50,0.69)$ & $(0.4,0.5,0.7,0.8)$ & $((0.33,0.5,0.67), 0.7,0.3)$ & 5.8 & {$[2,4]$} \\
\hline & $D_{5}$ & $(0.57,0.74,1.00)$ & $(0.2,0.3,0.6,0.6)$ & $((0.17,0.33,0.5), 0.4,0.6)$ & 7.0 & {$[4,6]$} \\
\hline
\end{tabular}

Step 1. The group decision matrices are obtained as in Table 1.

Step 2. Due to the ratings of $A_{1}, A_{2}, A_{3}$ given by DMs based on the one-mark system and the ratings of $A_{4}, A_{5}$, with the ten-mark system, we have

$$
\begin{array}{ll}
A_{j}^{\min }= \begin{cases}0, & \text { if } j \in N_{1} \\
{[0,0],} & \text { if } j \in N_{2} \\
(0,0,0), & \text { if } j \in N_{3} \\
(0,0,0,0), & \text { if } j \in N_{4} \\
((0,0,0), 0,1), & \text { if } j \in N_{5},\end{cases} \\
A_{j}^{\max }= \begin{cases}10, & \text { if } j \in N_{1} \\
{[10,10],} & \text { if } j \in N_{2} \\
(1,1,1), & \text { if } j \in N_{3} \\
(1,1,1,1), & \text { if } j \in N_{4} \\
((1,1,1), 1,0), & \text { if } j \in N_{5} .\end{cases}
\end{array}
$$

Obtain the aggregated ITFNs corresponding to the attribute vectors.

Step 2.1. By plugging (15) into (19)-(21) for benefit attributes (plugging (15) into (23), (24), and (21) for cost attributes), we can compute the Rsd, Rdd, and Rud of $x_{i j}^{k}$, which are shown in Table 2. Thereby the support set $\xi_{k j}$ and opposition set $\zeta_{k j}$ of $\boldsymbol{A}_{k j}$ can be derived.

Step 2.2. By (25)-(27), we can obtain the modes of $\xi_{k j}$ and $\zeta_{k j}$, which are listed in Table 3.
Step 2.3. Using (28) and (29), the Qst $\delta_{k j}$ and Qdt $\gamma_{k j}$ of $\boldsymbol{A}_{k j}$ can be constructed, and the results are presented in Table 3.

Step 2.4. Based on (30), the aggregated ITFNs of $\boldsymbol{A}_{k j}$ are also shown in Table 3.

Step 3. Determine the attribute weights.

Step 3.1. By (32) and (33), the intuitionistic triangular fuzzy PIS and NIS are defined as follows.

$$
\begin{aligned}
S^{+} & =\{((0.41,0.51,0.63),(0.13,0.24,0.34)), \\
& ((0.23,0.33,0.39),(0.18,0.29,0.39)), \\
& ((0.28,0.38,0.46),(0.19,0.27,0.36)), \\
& ((0.10,0.22,0.36),(0.18,0.32,0.44)), \\
& ((0.32,0.46,0.56),(0.13,0.24,0.35))\}, \\
S^{-} & =\{((0.35,0.42,0.50),(0.21,0.28,0.36)), \\
& ((0.13,0.22,0.33),(0.24,0.34,0.43)), \\
& ((0.07,0.18,0.29),(0.24,0.34,0.45)), \\
& ((0.00,0.16,0.29),(0.23,0.34,0.49)), \\
& ((0.22,0.32,0.44),(0.19,0.31,0.41))\} .
\end{aligned}
$$

Step 3.2. By (34) and (35), the similarity degrees $\vartheta_{k}^{+}$and $\vartheta_{k}^{-}(k=1,2,3,4)$ are calculated as follows.

$$
\begin{aligned}
\vartheta_{1}^{+} & =1-\left(0.097 w_{1}+0.062 w_{2}+0.051 w_{3}+0.004 w_{4}\right. \\
& \left.+0.026 w_{5}\right)
\end{aligned}
$$


TABLE 2: Rsd, Rdd and Rud of each attribute value.

\begin{tabular}{|c|c|c|c|c|c|c|}
\hline Providers & $\mathrm{DM}$ & $A_{1}$ & $A_{2}$ & $A_{3}$ & $A_{4}$ & $A_{5}$ \\
\hline \multirow[t]{5}{*}{$S_{1}$} & $D_{1}$ & $0.67,0.33,0.49$ & $0.50,0.50,1.00$ & $0.42,0.58,0.64$ & $0.66,0.34,0.52$ & $0.60,0.40,0.67$ \\
\hline & $D_{2}$ & $0.49,0.51,0.39$ & $0.58,0.42,0.74$ & $0.42,0.58,0.61$ & $0.46,0.54,0.85$ & $0.80,0.20,0.25$ \\
\hline & $D_{3}$ & $0.67,0.33,0.52$ & $0.53,0.47,0.90$ & $0.41,0.59,0.68$ & $0.28,0.72,0.39$ & $0.65,0.35,0.54$ \\
\hline & $D_{4}$ & $0.70,0.30,0.50$ & $0.48,0.52,0.90$ & $0.59,0.41,0.79$ & $0.19,0.81,0.24$ & $0.75,0.25,0.33$ \\
\hline & $D_{5}$ & $0.58,0.42,0.44$ & $0.28,0.720 .38$ & $0.67,0.33,0.80$ & $0.41,0.59,0.70$ & $0.45,0.55,0.82$ \\
\hline \multirow[t]{5}{*}{$S_{2}$} & $D_{1}$ & $0.64,0.36,0.49$ & $0.40,0.60,0.67$ & $0.70,0.30,0.58$ & $0.58,0.42,0.72$ & $0.55,0.45,0.82$ \\
\hline & $D_{2}$ & $0.57,0.43,0.43$ & $0.50,0.50,1.00$ & $0.42,0.58,0.61$ & $0.46,0.54,0.85$ & $0.65,0.35,0.54$ \\
\hline & $D_{3}$ & $0.82,0.18,0.60$ & $0.33,0.67,0.68$ & $0.69,0.31,0.56$ & $0.28,0.72,0.39$ & $0.45,0.55,0.82$ \\
\hline & $D_{4}$ & $0.54,0.46,0.41$ & $0.68,0.32,0.33$ & $0.45,0.55,0.76$ & $0.00,1.00,0.00$ & $0.80,0.20,0.25$ \\
\hline & $D_{5}$ & $0.83,0.17,0.60$ & $0.23,0.77,0.78$ & $0.63,0.37,0.89$ & $0.29,0.71,0.41$ & $0.35,0.65,0.54$ \\
\hline \multirow[t]{5}{*}{$S_{3}$} & $D_{1}$ & $0.66,0.34,0.50$ & $0.63,0.37,0.60$ & $0.15,0.85,0.17$ & $0.22,0.78,0.28$ & $0.70,0.30,0.43$ \\
\hline & $D_{2}$ & $0.58,0.42,0.44$ & $0.60,0.40,0.67$ & $0.42,0.58,0.61$ & $0.35,0.65,0.54$ & $0.70,0.30,0.43$ \\
\hline & $D_{3}$ & $0.71,0.29,0.55$ & $0.50,0.50,1.00$ & $0.14,0.86,0.16$ & $0.57,0.43,0.75$ & $0.80,0.20,0.25$ \\
\hline & $D_{4}$ & $0.77,0.23,0.56$ & $0.55,0.45,0.82$ & $0.44,0.56,0.70$ & $0.17,0.83,0.21$ & $0.60,0.40,0.67$ \\
\hline & $D_{5}$ & $0.54,0.46,0.43$ & $0.38,0.62,0.60$ & $0.56,0.44,0.91$ & $0.45,0.550 .82$ & $0.45,0.55,0.82$ \\
\hline \multirow[t]{5}{*}{$S_{4}$} & $D_{1}$ & $0.49,0.51,0.39$ & $0.35,0.65,0.54$ & $0.53,0.47,0.90$ & $0.51,0.49,0.96$ & $0.45,0.55,0.82$ \\
\hline & $D_{2}$ & $0.64,0.36,0.51$ & $0.33,0.67,0.48$ & $0.59,0.41,0.90$ & $0.19,0.81,0.24$ & $0.45,0.55,0.82$ \\
\hline & $D_{3}$ & $0.57,0.43,0.45$ & $0.28,0.72,0.38$ & $0.41,0.59,0.68$ & $0.61,0.39,0.64$ & $0.45,0.55,0.82$ \\
\hline & $D_{4}$ & $0.55,0.45,0.44$ & $0.60,0.40,0.67$ & $0.47,0.53,0.77$ & $0.42,0.58,0.72$ & $0.70,0.30,0.43$ \\
\hline & $D_{5}$ & $0.77,0.23,0.55$ & $0.43,0.57,0.74$ & $0.56,0.44,0.91$ & $0.30,0.70,0.43$ & $0.50,0.50,1.00$ \\
\hline
\end{tabular}

$$
\begin{aligned}
\vartheta_{2}^{+} & =1-\left(0.002 w_{1}+0.078 w_{2}+0.008 w_{3}+0.078 w_{4}\right. \\
& \left.+0.086 w_{5}\right) \\
\vartheta_{3}^{+} & =1-\left(0.040 w_{1}+0.030 w_{2}+0.165 w_{3}+0.043 w_{4}\right. \\
& \left.+0.026 w_{5}\right) \\
\vartheta_{4}^{+} & =1-\left(0.072 w_{1}+0.085 w_{2}+0.098 w_{3}+0.018 w_{4}\right. \\
& \left.+0.109 w_{5}\right) \\
\vartheta_{1}^{-} & =1-\left(0.014 w_{1}+0.038 w_{2}+0.141 w_{3}+0.079 w_{4}\right. \\
& \left.+0.114 w_{5}\right) \\
\vartheta_{2}^{-} & =1-\left(0.100 w_{1}+0.043 w_{2}+0.165 w_{3}+0.146 w_{4}\right. \\
& \left.+0.267 w_{5}\right) \\
\vartheta_{3}^{-} & =1-\left(0.060 w_{1}+0.087 w_{2}+0.003 w_{3}+0.166 w_{4}\right. \\
& \left.+0.323 w_{5}\right) \\
\vartheta_{4}^{-} & =1-\left(0.034 w_{1}+0.021 w_{2}+0.077 w_{3}+0.191 w_{4}\right. \\
& \left.+0.230 w_{5}\right)
\end{aligned}
$$

Step 3.3. By using (36), a multiple objective programming model is expressed as follows:

$\max$

$$
\begin{aligned}
& \left\{\vartheta_{1}^{+}-\vartheta_{1}^{-}=-0.084 w_{1}-0.023 w_{2}+0.089 w_{3}\right. \\
+ & \left.0.075 w_{4}+0.088 w_{5}\right\}
\end{aligned}
$$

$\max$

$$
\begin{aligned}
& \left\{\vartheta_{2}^{+}-\vartheta_{2}^{-}=0.098 w_{1}-0.035 w_{2}+0.156 w_{3}\right. \\
+ & \left.0.068 w_{4}+0.181 w_{5}\right\}
\end{aligned}
$$

$\max$

$$
\begin{aligned}
& \left\{\vartheta_{3}^{+}-\vartheta_{3}^{-}=0.020 w_{1}+0.058 w_{2}-0.162 w_{3}\right. \\
+ & \left.0.123 w_{4}+0.298 w_{5}\right\}
\end{aligned}
$$

$\max$

$$
\begin{aligned}
& \left\{\vartheta_{4}^{+}-\vartheta_{4}^{-}=-0.038 w_{1}-0.064 w_{2}-0.021 w_{3}\right. \\
+ & \left.0.172 w_{4}+0.122 w_{5}\right\}
\end{aligned}
$$

s.t. $\boldsymbol{w} \in \Omega$ 
TABLE 3: Mode $m\left\{\xi_{k j}\right\}, m\left\{\zeta_{k j}\right\}$, Qst $\delta_{k j}$, Qdt $\gamma_{k j}$ and aggregated ITFNs of attribute vectors.

\begin{tabular}{lcccccc}
\hline Sellers & Attributes & $m\left\{\xi_{k j}\right\}$ & $\xi_{k j}$ & $m\left\{\zeta_{k j}\right\}$ & $\zeta_{k j}$ & ITFNs \\
\hline$S_{1}$ & $A_{1}$ & 0.62 & $(0.49,0.62,0.70)$ & 0.38 & $(0.30,0.38,0.51)$ & $((0.35,0.44,0.49),(0.21,0.27,0.36))$ \\
& $A_{2}$ & 0.48 & $(0.28,0.48,0.58)$ & 0.52 & $(0.43,0.52,0.73)$ & $((0.16,0.28,0.34),(0.25,0.31,0.43))$ \\
& $A_{3}$ & 0.59 & $(0.50,0.59,0.83)$ & 0.41 & $(0.17,0.41,0.50)$ & $((0.35,0.41,0.58),(0.12,0.29,0.35))$ \\
& $A_{4}$ & 0.40 & $(0.19,0.40,0.66)$ & 0.60 & $(0.34,0.60,0.81)$ & $((0.10,0.22,0.36),(0.19,0.33,0.45))$ \\
& $A_{5}$ & 0.65 & $(0.45,0.65,0.80)$ & 0.35 & $(0.20,0.35,0.55)$ & $((0.32,0.46,0.56),(0.14,0.24,0.39))$ \\
\hline$S_{2}$ & $A_{1}$ & 0.68 & $(0.54,0.68,0.83)$ & 0.32 & $(0.17,0.32,0.46)$ & $((0.40,0.51,0.62),(0.12,0.24,0.34))$ \\
& $A_{2}$ & 0.42 & $(0.23,0.42,0.68)$ & 0.58 & $(0.33,0.58,0.78)$ & $((0.13,0.24,0.38),(0.18,0.33,0.44))$ \\
& $A_{3}$ & 0.71 & $(0.50,0.71,0.83)$ & 0.29 & $(0.17,0.29,0.50)$ & $((0.37,0.53,0.62),(0.12,0.22,0.37))$ \\
& $A_{4}$ & 0.67 & $(0.00,0.33,0.58)$ & 0.67 & $(0.42,0.67,1.00)$ & $((0.00,0.16,0.29),(0.21,0.33,0.50))$ \\
& $A_{5}$ & 0.44 & $(0.35,0.56,0.80)$ & 0.44 & $(0.20,0.44,0.65)$ & $((0.22,0.35,0.51),(0.13,0.28,0.41))$ \\
\hline$S_{3}$ & $A_{1}$ & 0.65 & $(0.54,0.65,0.77)$ & 0.35 & $(0.23,0.35,0.46)$ & $((0.40,0.48,0.57),(0.17,0.26,0.34))$ \\
& $A_{2}$ & 0.53 & $(0.38,0.53,0.63)$ & 0.47 & $(0.38,0.47,0.63)$ & $((0.24,0.34,0.40),(0.24,0.30,0.40)$ \\
& $A_{3}$ & 0.40 & $(0.17,0.40,0.67)$ & 0.60 & $(0.33,0.60,0.83)$ & $((0.09,0.22,0.37),(0.18,0.33,0.46))$ \\
& $A_{4}$ & 0.35 & $(0.17,0.35,0.57)$ & 0.65 & $(0.43,0.65,0.83)$ & $((0.09,0.19,0.31),(0.23,0.35,0.45))$ \\
& $A_{5}$ & 0.46 & $(0.32,0.46,0.56)$ & 0.24 & $(0.14,0.24,0.39)$ & $((0.32,0.46,0.56),(0.14,0.24,0.39))$ \\
\hline$S_{4}$ & $A_{1}$ & 0.60 & $(0.49,0.60,0.77)$ & 0.40 & $(0.23,0.40,0.51)$ & $((0.35,0.42,0.54),(0.16,0.28,0.35))$ \\
& $A_{2}$ & 0.39 & $(0.28,0.39,0.60)$ & 0.61 & $(0.40,0.61,0.73)$ & $((0.16,0.22,0.34),(0.23,0.35,0.41))$ \\
& $A_{3}$ & 0.54 & $(0.33,0.54,0.67)$ & 0.46 & $(0.33,0.46,0.67)$ & $((0.21,0.34,0.42),(0.21,0.29,0.42))$ \\
& $A_{4}$ & 0.41 & $(0.19,0.41,0.61)$ & 0.59 & $(0.39,0.59,0.81)$ & $((0.11,0.23,0.34),(0.22,0.33,0.45))$ \\
& $A_{5}$ & 0.50 & $(0.45,0.50,0.70)$ & 0.40 & $(0.30,0.40,0.55)$ & $((0.29,0.33,0.46),(0.20,0.32,0.36))$ \\
\hline
\end{tabular}

Step 3.4. By using (37), (43) can be changed into the following single objective linear programming model:

$$
\begin{array}{ll}
\max & \{x\} \\
\text { s.t. } & \left\{\begin{array}{l}
-0.084 w_{1}-0.020 w_{2}+0.089 w_{3}+0.075 w_{4}+0.088 w_{5} \geq x \\
0.098 w_{1}-0.030 w_{2}+0.154 w_{3}+0.068 w_{4}+0.181 w_{5} \geq x \\
0.020 w_{1}+0.058 w_{2}-0.162 w_{3}+0.123 w_{4}+0.298 w_{5} \geq x \\
-0.038 w_{1}-0.070 w_{2}-0.021 w_{3}+0.172 w_{4}+0.122 w_{5} \geq x \\
\boldsymbol{w} \in \Omega
\end{array}\right.
\end{array}
$$

Step 3.5. Applying Lingo 11.0, we have the attribute weight vector $\boldsymbol{w}=(0.162,0.212,0.214,0.200,0.212)$.

Step 4. By plugging $\boldsymbol{w}$ into (34) and (35), the similarity degrees $\vartheta_{k}^{+}$and $\vartheta_{k}^{-}$of alternative $S_{k}$ can be calculated as follows:

$$
\begin{aligned}
& \vartheta_{3}^{-}=0.869, \\
& \vartheta_{4}^{+}=0.923, \\
& \vartheta_{4}^{-}=0.886 .
\end{aligned}
$$

Step 5. Based on (37), the RCC of alternative $S_{k}$ is yielded as follows:

$$
\begin{aligned}
& R C C_{1}=0.509, \\
& R C C_{2}=0.526, \\
& R C C_{3}=0.519, \\
& R C C_{4}=0.510 .
\end{aligned}
$$

$$
\begin{aligned}
& \vartheta_{1}^{+}=0.954, \\
& \vartheta_{1}^{-}=0.919, \\
& \vartheta_{2}^{+}=0.948, \\
& \vartheta_{2}^{-}=0.854, \\
& \vartheta_{3}^{+}=0.938,
\end{aligned}
$$


Step 6. Since $R C C_{2}>R C C_{3}>R C C_{4}>R C C_{1}$, we can easily rank the preference order of the sellers as $S_{2}>S_{3}>S_{4}>S_{1}$. Hence, the best seller is $S_{2}$.

5.2. Comparison Analyses with Existing Methods Aggregated IF Information. Generally, the key focus of the heterogeneous MAGDM methods is how to aggregate the assessments taking the form of different data types, determine the weights of experts and attributes, and rank the preference order of alternatives. Here, we make detailed comparison analyses with some similar methods $[34,35,37]$ from the abovementioned key issues, which are shown in Table 4. Furthermore, the current methodology has the following superiorities.

(1) A comparison between the current methodology and Yue's method [34] is made. We consider attribute weight vector $\boldsymbol{w}=(0.4,0.2,0.4)$ and employ the former to evaluate the masses' satisfaction of three leaders used in [34]; the ranking of three suppliers is $S_{1}>S_{3}>S_{2}$ which is inconsistent with $S_{1}>S_{2}>S_{3}$ shown in [34]. Although the ranking is inconsistent, the best satisfactory leader is the same. Thus, the current methodology can well adapt to the special decision circumstance in [34]. However, the latter cannot deal with the heterogeneous MAGDM problems since it is only suitable for interval numbers. Moreover, in the former, the attribute weights are derived by using a multiple objective programming model that can avoid subjective randomness in the latter.

(2) A comparison between the current methodology and $\mathrm{Xu}$ et al. method [35] is made. Assume that $a=\left(\mu_{1}, v_{1}\right)$ and $b=\left(\mu_{2}, v_{2}\right)$ are two IFNs, $d(a, b)=(1 / 2)\left(\left|\mu_{1}-\mu_{2}\right|+\left|v_{1}-v_{2}\right|\right)$ is the distance of $a$ and $b$, and $\alpha^{+}=(1,0)$ and $\alpha^{-}=(0,1)$ are the largest and smallest IFN. Then, the former is applied to evaluate the cloud computing service provider used in [35]; the ranking of four suppliers is $S_{1}>S_{2}>S_{3}>S_{4}$ which is inconsistent with $S_{3}>S_{1}>S_{2}>S_{4}$ shown in [35]. Thus, the current methodology can well adapt to a special decision circumstance in [35]. The main reasons for the difference in the ranking are as follows. (i) The method for eliciting the satisfaction and dissatisfaction of DMs based on the TOPSIS idea in the current methodology is more complete than that in [35], because the former takes into account the two aspects of satisfaction and dissatisfaction in each element, while the latter considered only one aspect. (ii) Besides the rating values denoted by RNs, INs, TFNs, TrFNs, and LVs, the former can deal with more complicated rating values, including TIFNs, whereas the latter is only suitable for the HMAGDM problem with RNs, INs, TFNs, TrFNs, and LVs. Thus, the latter cannot solve the abovementioned example. (iii) In the former, the integrated information of experts is expressed by a TIFN, whereas that in the latter is an IFN. Hence, there are more opportunities resulting in a loss of information.

(3) A comparison between the current methodology and Wan et al. method [37] is made. We employ the former to evaluate the IT outsourcing service provider used in [37]; the ranking of four suppliers is $S_{3}>S_{2}>S_{1}>S_{4}$ which is consistent with $S_{3}>S_{2}>S_{1}>S_{4}$ shown in [37]. Thus, the current methodology can well adapt to a special decision circumstance in [37]. However, the current methodology is superior over the later in the following aspects. (i) In the former, the integrated information of experts on the same attribute is a TIFN, whereas that in the latter is an IVIFN. Hence, the current methodology can express vagueness information of reality more accurately and abundantly. (ii) The former takes into account the weights of experts by their consistency degree, whereas there is no consideration in the latter. So, the current methodology is more reasonable. (iii) The same as the above, the latter is only suitable for the HMAGDM problem with RNs, INs, TFNs, and TrFNs. Thus, the latter cannot solve the abovementioned example.

\subsection{Comparison Analyses with Existing HMAGDM Methods.} In this section, we compare the proposed method with other two methods for HMAGDM problems; one is the complex and dynamic MAGDM method developed by Dong et al. [15] that is an optimization-based approach, and the other is the GDM method based on integrating heterogeneous information introduced by Li [21] that is a direct approach. For simplicity, the comparative analysis methods are denoted as CD-GDM and IGI-GDM. The highlighted features of the proposed methods can be summarized as follows.

(1) During the initial phase, CD-GDM and IGI-GDM need to standardize the decision data, whereas there is no need for standardization in the proposed method which is relatively simple.

(2) The GDM matrix of the proposed method is an ITFN decision-making matrix containing ITFNs only which is easy to handle, whereas that of IGI-GDM remains a heterogeneous decision-making matrix which is difficult to deal with.

(3) In CD-GDM and IGI-GDM, the integrated information of experts is expressed by original decision data types and real number type which contains less information, while the integrated information of experts in the proposed method is represented by ITFNs which can express intuitively and describe satisfaction, dissatisfaction, and distribution of experts.

(4) The weights of attributes in IGI-GDM are given by decision makers in advance which are subjective; CD-GDM determine the weights of attributes by nonlinear programming model, whereas they construct a multiple objective linear programming model to establish attributes' weights. Thus the proposed method is more objective and effective.

\section{Conclusions}

In this paper, we put forward a new aggregation approach to solve such heterogeneous MAGDM problems in which the weights of the attributes are incompletely known. The key features of the proposed method are listed as follows: (1) a new similarity measure of ITFNs is proposed; (2) a new general approach to aggregating decision information into ITFNs is proposed. It can not only accommodate more complicated data of types, including INs, TFNs, TrFNs, and TIFNs, but also take importance of experts into account; (3) a new multiple objective mathematical programming model is developed for determining the attribute weights objectively under intuitionistic triangular fuzzy environment; (4) a new method is presented to solve heterogeneous MAGDM problems, which considers fully the indeterminacy of the 


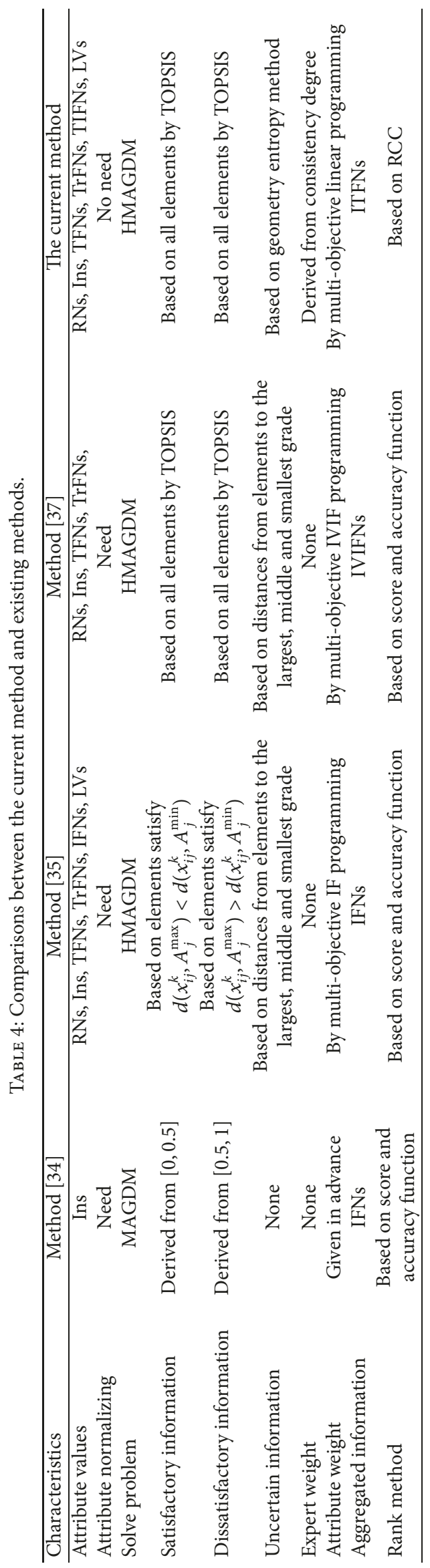


DMs in the assessment; thus the final decision results derived by the proposed method are more reasonable. Additionally, the proposed method can be also appropriate for the complex multiattribute large-group decision-making problems [51]. Future research will extend the developed method to heterogeneous MAGDM with complete unknown weight information under complex fuzzy environment. Meanwhile, as the scale of group increases and the decision makers have different backgrounds and levels of knowledge, it is difficult to achieve consensus among decision makers $[52,53]$. Therefore, it will be very interesting in future studies to discuss the consensus reaching mechanism in the large-scale HMAGDM.

\section{Data Availability}

We state that the data used to support the findings of this study are included within the article.

\section{Conflicts of Interest}

The authors declare that they have no conflicts of interest.

\section{Acknowledgments}

This work was supported by the National Natural Science Foundation of China (Nos. 61602219, 71740021, 11861034, and 11461030), the Colleges Humanities Social Science Research Project of Jiangxi Province of China (No. GL162047) and the Science and Technology Project of Jiangxi Province of China (No. GJJ188412), the Natural Science Foundation of Jiangxi Province of China (No. 20192BAB207012) and the "Thirteen Five" Programming Project of Jiangxi Province Social Science (No. 18GL13).

\section{References}

[1] Z. L. Yue, "TOPSIS-based group decision-making methodology in intuitionistic fuzzy setting," Information Sciences, vol. 277, pp. 141-153, 2014.

[2] P. Liu and F. Teng, "Multiple criteria decision making method based on normal interval-valued intuitionistic fuzzy generalized aggregation operator," Complexity, vol. 21, no. 5, pp. 277290, 2016

[3] P. Liu and F. Teng, "An extended TODIM method for multiple attribute group decision-making based on 2-dimension uncertain linguistic variable," Complexity, vol. 21, no. 5, pp. 20-30, 2016.

[4] X. Chen, H. Zhang, and Y. Dong, "The fusion process with heterogeneous preference structures in group decision making: a survey," Information Fusion, vol. 24, pp. 72-83, 2015.

[5] I. Igoulalene, L. Benyoucef, and M. K. Tiwari, "Novel fuzzy hybrid multi-criteria group decision making approaches for the strategic supplier selection problem," Expert Systems with Applications, vol. 42, no. 7, pp. 3342-3346, 2015.

[6] C. De Maio, G. Fenza, V. Loia, F. Orciuoli, and E. HerreraViedma, "A framework for context-aware heterogeneous group decision making in business processes," Knowledge-Based Systems, vol. 102, pp. 39-50, 2016.
[7] C. Yue, "A projection-based approach to software quality evaluation from the users perspectives," International Journal of Machine Learning and Cybernetics, pp. 1-13, 2018.

[8] C. Yue, "A normalized projection-based group decision-making method with heterogeneous decision information and application to software development effort assessment," Applied Intelligence, 2019.

[9] C. Yue, "Normalization of attribute values with interval information in group decision-making setting: With an application to software quality evaluation," Journal of Experimental \& Theoretical Artificial Intelligence, vol. 31, no. 3, pp. 475-492, 2019.

[10] Z. Yue and Y. Jia, "A group decision making model with hybrid intuitionistic fuzzy information," Computers \& Industrial Engineering, vol. 87, pp. 202-212, 2015.

[11] F. Herrera, L. Martínez, and P. J. Sánchez, "Managing nonhomogeneous information in group decision making," European Journal of Operational Research, vol. 166, no. 1, pp. 115-132, 2005.

[12] Y. Dong, Y. Xu, and S. Yu, "Linguistic multiperson decision making based on the use of multiple preference relations," Fuzzy Sets and Systems, vol. 160, no. 5, pp. 603-623, 2009.

[13] X. Wang and J. Cai, "A group decision-making model based on distance-based VIKOR with incomplete heterogeneous information and its application to emergency supplier selection," Kybernetes, vol. 46, no. 3, pp. 501-529, 2017.

[14] H. Zhang, Y. Dong, and X. Chen, "The 2-rank consensus reaching model in the multigranular linguistic multiple-attribute group decision-making," IEEE Transactions on Systems, Man, and Cybernetics: Systems, vol. 48, no. 12, pp. 2080-2094, 2018.

[15] Y. Dong, H. Zhang, and E. Herrera-Viedma, "Consensus reaching model in the complex and dynamic MAGDM problem," Knowledge-Based Systems, vol. 106, pp. 206-219, 2016.

[16] W. Zhang, Y. Ju, X. Liu, and M. Giannakis, "A mathematical programming-based method for heterogeneous multicriteria group decision analysis with aspirations and incomplete preference information," Computers \& Industrial Engineering, vol. 113, pp. 541-557, 2017.

[17] J. Dong and S. Wan, "A PROMETHEE-FLP method for heterogeneous multi-attributes group decision making," IEEE Access, vol. 6, pp. 46656-46667, 2018.

[18] Z. Yue and Y. Jia, "A direct projection-based group decisionmaking methodology with crisp values and interval data," Soft Computing, vol. 21, no. 9, pp. 2395-2405, 2017.

[19] C. Yue, "Normalized projection approach to group decisionmaking with hybrid decision information," International Journal of Machine Learning and Cybernetics, vol. 9, no. 8, pp. 13651375, 2018.

[20] S. Das, D. Guha, and R. Mesiar, "Extended bonferroni mean under intuitionistic fuzzy environment based on a strict $\mathrm{t}$ conorm," IEEE Transactions on Systems, Man, and Cybernetics: Systems, vol. 47, no. 8, pp. 2083-2099, 2017.

[21] G. Li, G. Kou, and Y. Peng, "A group decision making model for integrating heterogeneous information," IEEE Transactions on Systems, Man, and Cybernetics: Systems, vol. 48, no. 6, pp. 982992, 2018.

[22] K. T. Atanassov, "Intuitionistic fuzzy sets," Fuzzy Sets and Systems, vol. 20, no. 1, pp. 87-96, 1986.

[23] Z. Xu, "Intuitionistic fuzzy aggregation operators," IEEE Transactions on Fuzzy Systems, vol. 15, no. 6, pp. 1179-1187, 2007.

[24] K. Atanassov and G. Gargov, "Interval valued intuitionistic fuzzy sets," Fuzzy Sets and Systems, vol. 31, no. 3, pp. 343-349, 1989. 
[25] J. Xu, Y. Zhong, and S. Wan, "Incentive adaptive trust model based on integrated intuitionistic fuzzy information," Journal of Electronics and Information Technology, vol. 38, no. 4, pp. 803810, 2016.

[26] C. Yue, "An interval-valued intuitionistic fuzzy projectionbased approach and application to evaluating knowledge transfer effectiveness," Neural Computing and Applications, 2018.

[27] C. Yue, "An intuitionistic fuzzy projection-based approach and application to software quality evaluation," Soft Computing, 2019.

[28] Z. L. Yue, "The comprehensive evaluation of urban environmental quality based on intuitionistic fuzzy set," Mathematics in Practice and Theory, vol. 38, no. 8, pp. 51-58, 2008.

[29] Z. L. Yue, Y. Y. Jia, and X. L. Huang, "Multiple attribute decision making method based on intuitionistic fuzzy set," in Proceedings of the Second International Conference on Intelligent Information Management Systems and Technology, vol. 24, pp. 137-140, 2007.

[30] Z. L. Yue, Y. Y. Jia, and G. D. Ye, "An approach for multiple attribute group decision making based on intuitionistic fuzzy information," International Journal of Uncertainty, Fuzziness and Knowledge-Based System, vol. 17, no. 3, pp. 317-332, 2009.

[31] Z. Yue, "Aggregating crisp values into intuitionistic fuzzy number for group decision making," Applied Mathematical Modelling: Simulation and Computation for Engineering and Environmental Systems, vol. 38, no. 11-12, pp. 2969-2982, 2014.

[32] Z. L. Yue, "An approach to aggregating interval numbers into interval-valued intuitionistic fuzzy information for group decision making," Expert Systems with Applications, vol. 38, no. 5, pp. 6333-6338, 2011.

[33] Z. L. Yue and Y. Y. Jia, "A method to aggregate crisp values into interval-valued intuitionistic fuzzy information for group decision making," Applied Soft Computing, vol. 13, no. 5, pp. 2304-2317, 2013.

[34] Z. Yue, "A group decision making approach based on aggregating interval data into interval-valued intuitionistic fuzzy information," Applied Mathematical Modelling, vol. 38, no. 2, pp. 683-698, 2014.

[35] J. Xu, S.-P. Wan, and J.-Y. Dong, "Aggregating decision information into Atanassov's intuitionistic fuzzy numbers for heterogeneous multi-attribute group decision making," Applied Soft Computing, vol. 41, pp. 331-351, 2016.

[36] C. L. Hwang and K. Yoon, Multiple Attribute Decision Making: Methods and Applications, Springer, Heidelberg, Germany, 1981.

[37] S. Wan, J. Xu, and J. Dong, "Aggregating decision information into interval-valued intuitionistic fuzzy numbers for heterogeneous multi-attribute group decision making," KnowledgeBased Systems, vol. 113, pp. 155-170, 2016.

[38] F. Liu and X. H. Yuan, "Fuzzy number intuitionistic fuzzy set," Fuzzy Systems and Mathematics, vol. 21, no. 1, pp. 88-91, 2007.

[39] M. H. Shu, C. H. Cheng, and J. R. Chang, "Using intuitionistic fuzzy sets for fault-tree analysis on printed circuit board assembly," Microelectronics Reliability, vol. 46, no. 12, pp. 21392148, 2006.

[40] X. F. Wang, "Fuzzy number intuitionistic fuzzy geometric aggregation operators and their application to decision making," Control and Decision, vol. 10, no. 2, pp. 104-111, 2008.

[41] G. W. Wei, "Induced fuzzy number intuitionistic fuzzy ordered weighted averaging (I-FIFOWA) operator and its application to group decision making," Chinese Journal of Management, vol. 7, no. 6, pp. 903-908, 2010.
[42] Y. Gao, D. Q. Zhou, C. C. Liu, and L. Zhang, “Triangular fuzzy number intuitionistic fuzzy aggregation operators and their application based on interaction," Systems Engineering Theory \& Practice, vol. 32, no. 9, pp. 1964-1972, 2012.

[43] S. Yu and Z. Xu, "Aggregation and decision making using intuitionistic multiplicative triangular fuzzy information," Journal of Systems Science and Systems Engineering, vol. 23, no. 1, pp. 2038, 2014.

[44] Q. Qin, F. Liang, L. Li, Y.-W. Chen, and G.-F. Yu, "A TODIMbased multi-criteria group decision making with triangular intuitionistic fuzzy numbers," Applied Soft Computing, vol. 55, pp. 93-107, 2017.

[45] A. C. Kutlu and M. Ekmekçioğlu, "Fuzzy failure modes and effects analysis by using fuzzy TOPSIS-based fuzzy AHP," Expert Systems with Applications, vol. 39, no. 1, pp. 61-67, 2012.

[46] E. Szmidt and J. Kacprzyk, "Entropy for intuitionistic fuzzy sets," Fuzzy Sets and Systems, vol. 118, no. 3, pp. 467-477, 2001.

[47] T. Y. Chen and C. H. Li, "Determining objective weights with intuitionistic fuzzy entropy measures: a comparative analysis," Information Sciences, vol. 180, no. 21, pp. 4207-4222, 2010.

[48] B. Farhadinia, "A theoretical development on the entropy of interval-valued fuzzy sets based on the intuitionistic distance and its relationship with similarity measure," Knowledge-Based Systems, vol. 39, pp. 79-84, 2013.

[49] C.-B. Cheng, "Group opinion aggregationbased on a grading process: a method for constructing triangular fuzzy numbers," Computers \& Mathematics with Applications, vol. 48, no. 10-11, pp. 1619-1632, 2004.

[50] X. Li and X. Chen, "Multi-criteria group decision making based on trapezoidal intuitionistic fuzzy information," Applied Soft Computing, vol. 30, pp. 454-461, 2015.

[51] Á. Labella, Y. Liu, R. M. Rodríguez, and L. Martínez, "Analyzing the performance of classical consensus models in large scale group decision making: A comparative study," Applied Soft Computing, vol. 67, pp. 677-690, 2018.

[52] H. Zhang, Y. Dong, I. Palomares-Carrascosa, and H. Zhou, "Failure mode and effect analysis in a linguistic context: a consensus-based multiattribute group decision-making approach," IEEE Transactions on Reliability, vol. 68, no. 2, pp. 566-582, 2018.

[53] H. Zhang, Y. Dong, F. Chiclana, and S. Yu, "Consensus efficiency in group decision making: A comprehensive comparative study and its optimal design," European Journal of Operational Research, vol. 275, no. 2, pp. 580-598, 2019. 


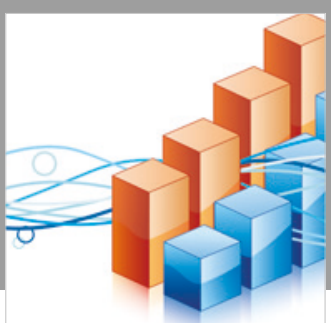

Advances in

Operations Research

\section{-n-m}
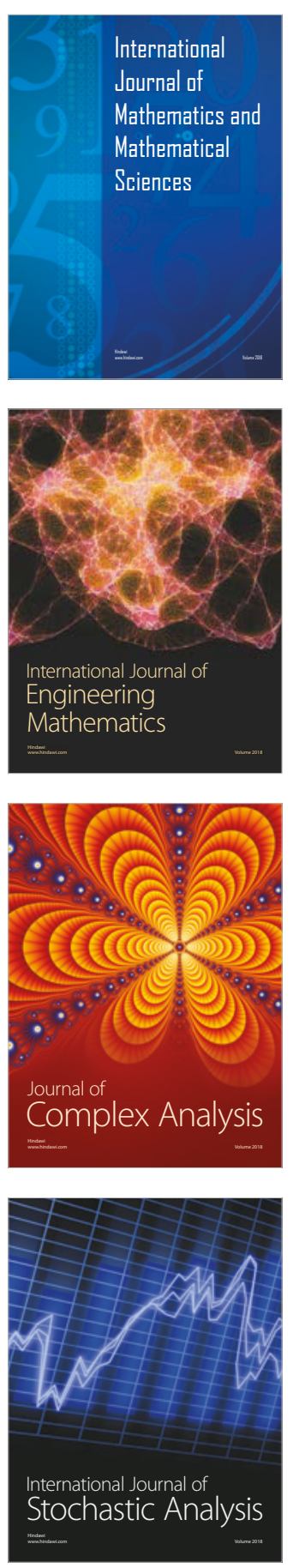
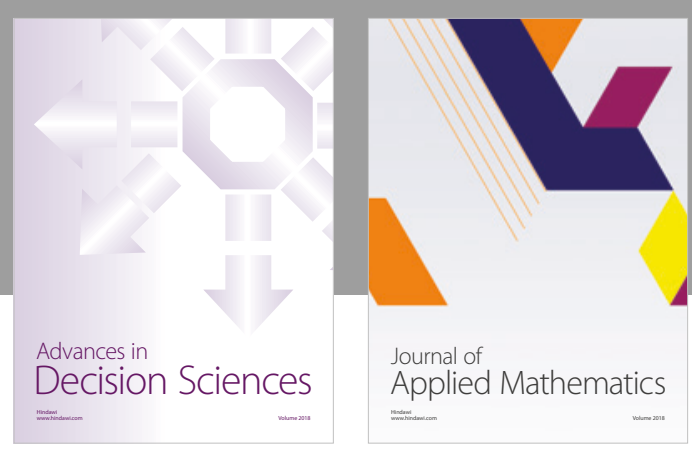

Journal of

Applied Mathematics
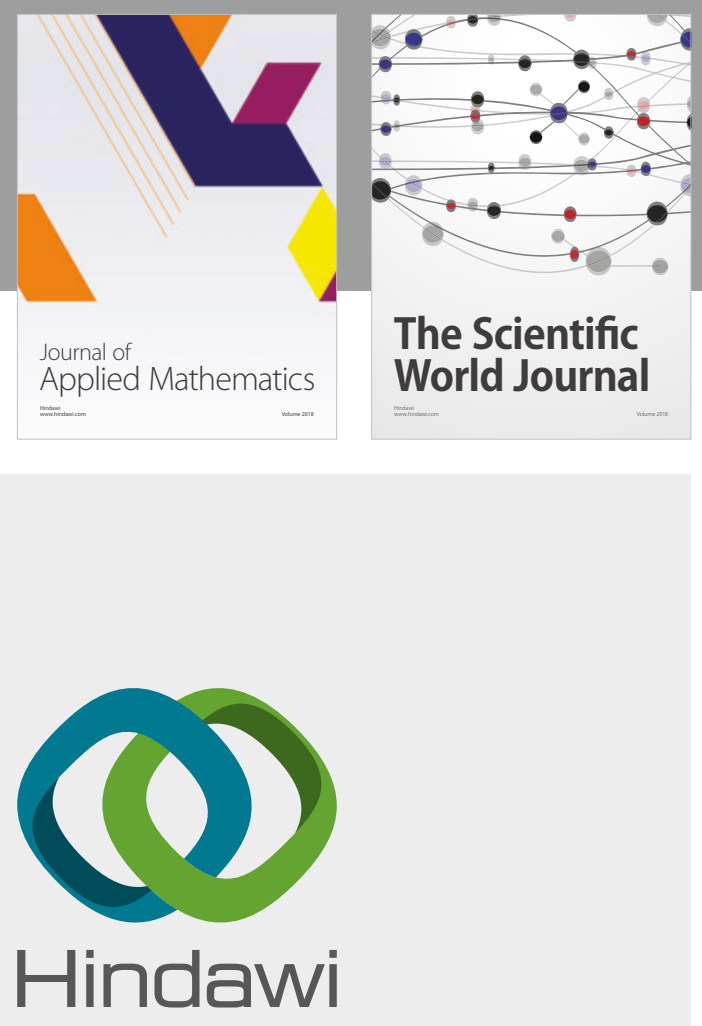

Submit your manuscripts at

www.hindawi.com

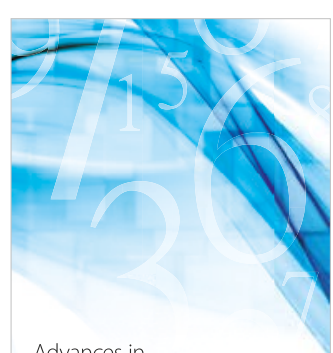

Advances in
Numerical Analysis
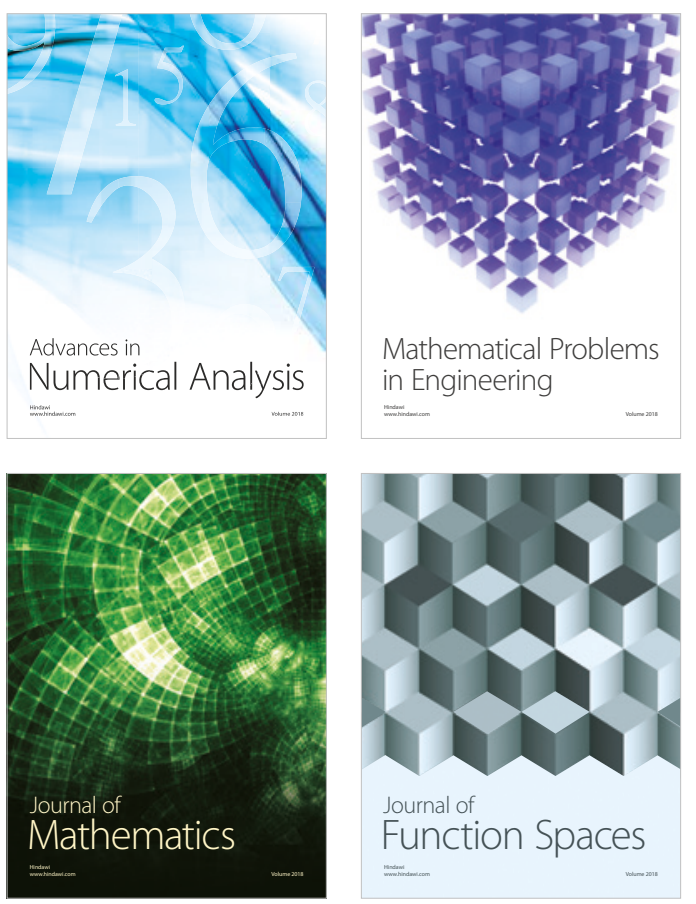

Mathematical Problems in Engineering

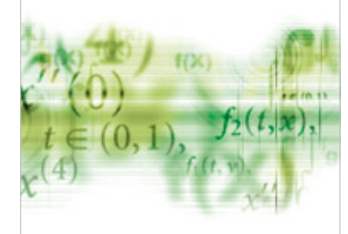

International Journal of

Differential Equations

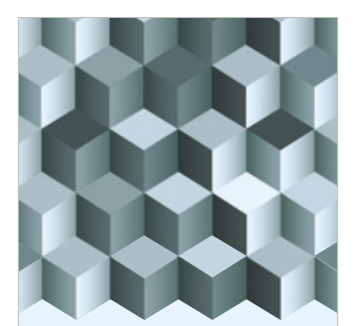

Journal of

Function Spaces
The Scientific

World Journal

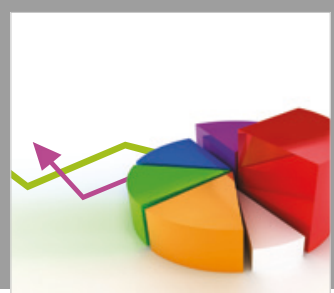

Journal of

Probability and Statistics
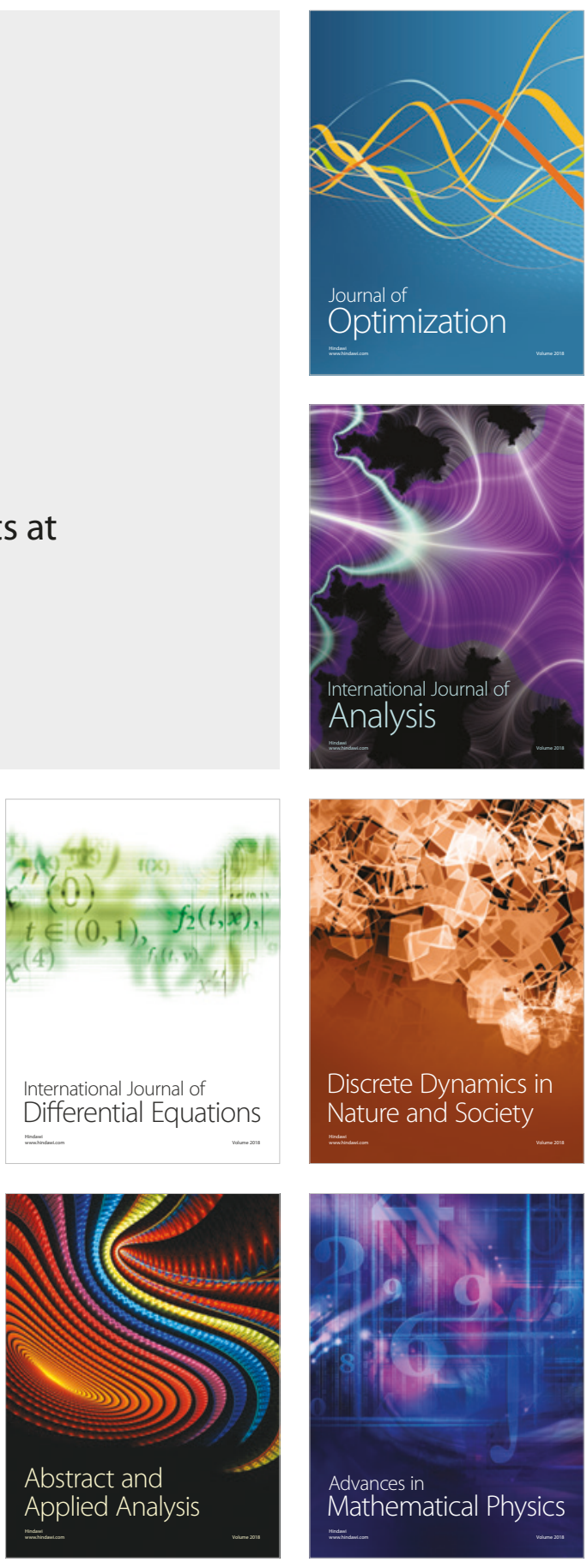